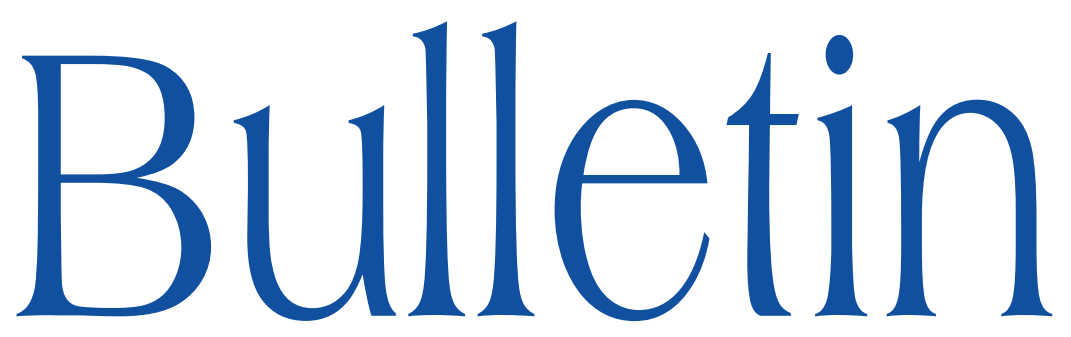

de la SOCIÉTÉ MATHÉMATIQUE DE FRANCE

\title{
ESTIMÉES POUR LES VALUATIONS $p$-ADIQUES DES VALEURS PROPRES DES OPÉRATEURS DE HECKE
}

\section{Vincent Lafforgue}

\section{Tome 139}

Fascicule 4 


\title{
ESTIMÉES POUR LES VALUATIONS $p$-ADIQUES DES VALEURS PROPRES DES OPÉRATEURS DE HECKE
}

\author{
PAR VINCENT LAFFORGUE
}

\begin{abstract}
RÉSumÉ. - Pour les formes automorphes cuspidales sur les corps de fonctions et pour les formes automorphes cuspidales cohomologiques sur les corps de nombres, on donne des estimées pour les valuations $p$-adiques des valeurs propres des opérateurs de Hecke. Dans le cas des corps de nombres, ces estimées correspondent aux estimées de KatzMazur par les conjectures de Langlands.

Abstract (Estimates for p-adic valuations of Hecke operator eigenvalues)

For cuspidal automorphic forms on function fields and for cohomological cuspidal automorphic forms on number fields we give estimates for the $p$-adic valuations of eigenvalues of Hecke operators. In the case of number fields, these estimates correspond to Katz-Mazur estimates by the Langlands conjectures.
\end{abstract}

Soit $X$ une variété projective lisse sur $\mathbb{Q}$ et $p$ un nombre premier tel que $X$ ait bonne réduction en $p$. Dans [14], Deligne montre que le polynôme caractéristique de l'action du Frobenius géométrique en $p$ sur la cohomologie $\ell$-adique $H^{i}\left(X_{\overline{\mathbb{Q}}}, \mathbb{Q}_{l}\right)$ a ses coefficients dans $\mathbb{Z}$ et est indépendant du nombre premier auxiliaire $\ell \neq p$. De plus ses valeurs propres sont des entiers algébriques qui vérifient les estimées suivantes :

Texte reçu le 16 janvier 2009, accepté le 14 janvier 2010.

Vincent Lafforgue, Laboratoire de Mathématiques, Analyse, Probabilités, Modélisation, Orléans (MAPMO), UMR CNRS 6628, Université d'Orléans, Rue de Chartres, B.P. 6759 45067 Orléans cedex 2, et Institut de Mathématiques de Jussieu, Equipe d'Algèbres d'Opérateurs, Université Paris 7 Denis Diderot, Etage 7, bureau E04, 175, rue de Chevaleret, 75013 Paris - E-mail : vlafforg@math.jussieu.fr

Classification mathématique par sujets (2000). - 11F70, 11F85.

Mots clefs. - Formes automorphes, opérateurs de Hecke, corps p-adiques, motifs. 
- pour tous les plongements de $\overline{\mathbb{Q}}$ dans $\mathbb{C}$, leurs images ont pour norme $p^{i / 2}$ (conjectures de Weil, voir [14])

- pour tous les plongements de $\overline{\mathbb{Q}}$ dans $\overline{\mathbb{Q}}_{\ell}$ avec $\ell \neq p$, leurs images ont pour norme 1 (par construction).

Katz et Mazur ont donné dans [31, 32] des estimées pour les valuations des images dans $\overline{\mathbb{Q}}_{p}$ des valeurs propres du Frobenius géométrique. Elles affirment que le polygone de Newton $p$-adique du polynôme caractéristique du Frobenius géométrique est au-dessus du polygone convexe associé à la filtration de Hodge de la cohomologie de de Rham de $X$. Dans la proposition 4.3.3. de [18] (voir aussi la remarque 4.4.6 de [21], [12] et [25]) Fontaine montre que ces estimées sont encore vérifiées dans le cadre plus général d'un motif $M$ sur un corps nombre $F$ à coefficients dans un corps de nombres $E$, pour toute place finie $v$ de $F$ où $M$ est non ramifié. Dans le paragraphe 4 nous rappelons cet argument, qui repose sur la faible admissibilité du $\varphi$-module filtré associé à la réalisation cristalline de $M$ en la place $v$.

Le but de cet article est de montrer de telles estimées $p$-adiques pour les formes automorphes. Plus précisément soit $\pi$ une forme automorphe cuspidale pour un groupe réductif déployé $G$ sur un corps global $F$. Soit $v$ une place finie de $F$ où $\pi$ est non ramifiée. Le paramètre de Satake de $\pi$ en $v$ est une classe de conjugaison $c_{\pi, v} \in \hat{G}(\mathbb{C})$ (en désignant par $\hat{G}$ le groupe dual de Langlands). On conjecture que $c_{\pi, v}$ est en fait une classe de conjugaison dans $\hat{G}(\overline{\mathbb{Q}})$ lorsque $\pi$ est algébrique (voir [11] pour le cas de $G L_{r}$ ) et on le sait au moins dans les deux cas suivants

- i) $F$ est un corps de fonctions,

- ii) $F$ est un corps de nombres et $\pi$ est cohomologique en les places infinies.

Soit $\mathfrak{p}$ une place dans $\overline{\mathbb{Q}}$ au-dessus de la caractéristique de $v$. La valuation associée à $\mathfrak{p}$ induit une application $N_{\mathfrak{p}}$ de l'ensemble des classes de conjugaison dans $\hat{G}(\overline{\mathbb{Q}})$ dans la chambre de Weyl positive $P_{\mathbb{R}}^{+}$qui dans le cas où $G=G L_{r}$ est le polygone de Newton $\mathfrak{p}$-adique du polynôme caractéristique. On possède dans $P_{\mathbb{R}}^{+}$la relation d'ordre partiel suivante $: \mu \leq \lambda$ si $\mu$ est dans l'enveloppe convexe des $w(\lambda)$ pour $w$ dans le groupe de Weyl ou de facon équivalente si $\lambda-\mu$ est une combinaison à coefficients dans $\mathbb{R}_{+}$des coracines positives de $\hat{G}$. Dans le cas où $G=G L_{r}$ on a $\mu \leq \lambda$ si et seulement si le polygone associé à $\mu$ est au-dessus du polygone associé à $\lambda$ et a les mêmes extrémités.

On montre alors

- dans le cas i) : $N_{\mathfrak{p}}\left(c_{\pi, v}\right) \leq \rho$ (voir la proposition 2.1)

- dans le cas ii) : $N_{\mathfrak{p}}\left(c_{\pi, v}\right) \leq \rho+\mu$ où $\mu$ dépend de façon explicite de $\mathfrak{p}$ et des caractères infinitésimaux de $\pi$ en les places infinies (voir la proposition 3.1).

TOME $139-2011-\mathrm{N}^{\mathrm{O}} 4$ 
La démonstration utilise les corollaires 1.2 et 1.4, qui résultent eux-mêmes d'une forme « entière » de l'isomorphisme de Satake figurant dans [23] et rappelée dans le premier paragraphe. Les mêmes idées apparaissent dans [34] et dans [13].

Dans le cas i) et pour $G=G L_{r}$, grâce à la correspondance de Langlands pour $G L_{r}$ montrée par Laurent Lafforgue [28], ces estimées ont pour conséquence des estimées sur les valuations $p$-adiques des valeurs propres de Frobenius géométrique pour les représentations galoisiennes irréductibles. Nous montrons que ces estimées sont optimales. Elles complètent les estimées conjecturées par Deligne dans [15] et celles montrées par Laurent Lafforgue dans [28].

Dans le cas ii) ces estimées sont équivalentes, pour un motif correspondant à une forme automorphe cuspidale cohomologique pour $G L_{r}$, aux estimées de Katz-Mazur sous la forme générale montrée par Fontaine que nous avons évoquée ci-dessus. En fait dans le cas ii), ces estimées étaient déjà connues, ainsi que le fait qu'elles correspondent aux estimées de Katz-Mazur. D'abord la question est posée dans la conjecture 4.8 de [11] et l'idée d'utiliser la cohomologie entière est indiquée dans les phrases suivantes (voir aussi le corollaire 5.2 de [11] pour une méthode différente). Dans le cas d'un système local trivial ces estimées sont établies dans [12] ainsi que le fait qu'elles correspondent exactement aux estimées de Katz-Mazur. Le cas d'un système local arbitraire est laissé en exercice (exercice 3.11 de [12]) et traité essentiellement dans [24, 25, 30].

Pour simplifier nous ne considérons dans cet article que des groupes déployés. Le cas des groupes non déployés et des places ramifiées est un peu discuté dans le paragraphe 5, en relation avec les résultats de Jean-François Dat [13].

Je remercie beaucoup Laurent Clozel, Jean-François Dat et Alain Genestier pour des discussions qui ont permis d'améliorer cet article. Je remercie le rapporteur pour ses remarques. De plus cette introduction est largement inspirée par le résumé inclus dans le rapport.

\section{Rappels sur l'isomorphisme de Satake}

Les rappels qui suivent sont extraits de l'article de Gross [23]. On renvoie aussi à [9] pour l'isomorphisme de Satake. Soit $K$ un corps local non archimédien et $\vartheta_{K}$ son anneau d'entiers. On note $\pi$ une uniformisante de $K, q$ le cardinal du corps résiduel et $p$ sa caractéristique. Soit $G$ un groupe réductif déployé sur $\theta_{K}$. On fixe un tore maximal déployé et un Borel le contenant : $T \subset B \subset G$.

On note $X_{*}(T)=\operatorname{Hom}\left(\mathbb{G}_{m}, T\right)$ et $X^{*}(T)=\operatorname{Hom}\left(T, \mathbb{G}_{m}\right)$ les ensembles des copoids et des poids de $G$.

On note $\hat{G}$ le groupe dual de $G$ (qui est un groupe réductif complexe) et $\hat{T}$ un tore maximal de $\hat{G}$ de sorte que $X^{*}(\hat{T})=X_{*}(T)$ et $X_{*}(\hat{T})=X^{*}(T)$. On 
note $\Phi^{+} \subset X^{*}(T)$ l'ensemble des racines positives et $\hat{\Phi}^{+} \subset X^{*}(\hat{T})$ l'ensemble des racines positives de $\hat{G}$ correspondant à $\Phi^{+}$. On note $W$ le groupe de Weyl de $G$, qui est aussi celui de $\hat{G}$. On note $w_{0}$ l'élément le plus long de $W$ (qui envoie la chambre de Weyl sur son opposé).

L'ensemble des poids dominants de $G$, qui est aussi l'ensemble des copoids dominants de $\hat{G}$, est

$$
P^{+}=\left\{\nu \in X_{*}(\hat{T}),\langle\beta, \nu\rangle \geq 0 \text { pour tout } \beta \in \hat{\Phi}^{+}\right\} .
$$

On introduit aussi la chambre de Weyl positive

$$
P_{\mathbb{R}}^{+}=\left\{\nu \in X_{*}(\hat{T}) \otimes_{\mathbb{Z}} \mathbb{R},\langle\beta, \nu\rangle \geq 0 \text { pour tout } \beta \in \hat{\Phi}^{+}\right\} .
$$

On a une identification $\left(X_{*}(\hat{T}) \otimes_{\mathbb{Z}} \mathbb{R}\right) / W=P_{\mathbb{R}}^{+}$et pour $\lambda, \mu \in P_{\mathbb{R}}^{+}$, on écrit $\mu \leq \lambda$ si l'une des assertions équivalentes suivantes est vérifiée :

- $\mu$ appartient à l'enveloppe convexe des $w(\lambda)$ dans $X_{*}(\hat{T}) \otimes_{\mathbb{Z}} \mathbb{R}$, pour $w \in$ $W$,

$-\lambda-\mu$ est une combinaison à coefficients dans $\mathbb{R}_{+}$des coracines positives de $\hat{G}$.

L'ensemble des poids dominants de $\hat{G}$, qui est aussi l'ensemble des copoids dominants de $G$, est

$$
\hat{P}^{+}=\left\{\lambda \in X_{*}(T),\langle\lambda, \alpha\rangle \geq 0 \text { pour tout } \alpha \in \Phi^{+}\right\} .
$$

Pour $\lambda, \mu \in \hat{P}^{+}$on écrit $\mu \leq \lambda$ si $\lambda-\mu$ est une somme de coracines positives de $G$.

Soit $d g$ la mesure de Haar sur $G(K)$ telle que $G\left(\theta_{K}\right)$ ait pour volume 1 . On note $\mathcal{H}$ l'algèbre de Hecke, formée des fonctions $G\left(\theta_{K}\right)$-bi-invariantes à support compact sur $G(K)$ à valeurs dans $\mathbb{Z}$, munie du produit de convolution $f . g(y)=$ $\int_{G(K)} f(x) g\left(x^{-1} y\right) d x$. Pour tout cocaractère $\lambda \in X_{*}(T)$ l'image de $\lambda(\pi) \in$ $T(K)$ dans $T(K) / T\left(\theta_{K}\right)$ ne dépend pas du choix de $\pi$. De plus $G(K)$ est la réunion disjointe des doubles classes $G\left(\theta_{K}\right) \lambda(\pi) G\left(\theta_{K}\right)$ quand $\lambda$ parcourt $\hat{P}^{+}$. Pour $\lambda \in \hat{P}^{+}$on note $c_{\lambda} \in \mathcal{H}$ la fonction caractéristique de $G\left(\theta_{K}\right) \lambda(\pi) G\left(\theta_{K}\right)$, de sorte que $\left(c_{\lambda}\right)_{\lambda \in \hat{P}^{+}}$forme une base de $\mathcal{H}$ sur $\mathbb{Z}$.

On note $R(\hat{G})$ l'anneau des représentations de $\hat{G}$, qui est le $\mathbb{Z}$-module libre engendré par les représentations irréductibles de $\hat{G}$, et dont la multiplication est le produit tensoriel des représentations. Pour toute représentation $V$ de $\hat{G}$, on note $[V]$ l'élément correspondant de $R(\hat{G})$.

L'isomorphisme de Satake est un isomorphisme de $\mathbb{Z}\left[q^{\frac{1}{2}}, q^{-\frac{1}{2}}\right]$-algèbres

$$
\mathcal{H} \otimes_{\mathbb{Z}} \mathbb{Z}\left[q^{\frac{1}{2}}, q^{-\frac{1}{2}}\right] \stackrel{\leftrightarrow}{\rightarrow} R(\hat{G}) \otimes_{\mathbb{Z}} \mathbb{Z}\left[q^{\frac{1}{2}}, q^{-\frac{1}{2}}\right]
$$

dont la définition est rappelée dans la proposition 3.6 de [23].

Le point essentiel pour nous est la proposition suivante, qui figure comme remarque dans [23] après la proposition 3.6. 
Pour tout $\lambda \in \hat{P}^{+}$on note $V_{\lambda}$ la représentation irréductible de $\hat{G}$ de plus haut poids $\lambda$, de sorte que $\left(\left[V_{\lambda}\right]\right)_{\lambda \in \hat{P}^{+}}$est une base de $R(\hat{G})$ sur $\mathbb{Z}$. On note

$$
2 \rho=\sum_{\alpha \in \Phi^{+}} \alpha \in X^{*}(T)=X_{*}(\hat{T}) .
$$

Proposition 1.1. - (23]) Il existe des entiers $b_{\lambda}(\mu) \in \mathbb{Z}$ pour $\lambda, \mu \in \hat{P}^{+}$tels que pour tout $\lambda \in \hat{P}^{+}$,

$$
\phi\left(c_{\lambda}\right)=q^{\langle\lambda, \rho\rangle}\left[V_{\lambda}\right]+\sum_{\mu<\lambda} b_{\lambda}(\mu)\left(q^{\langle\mu, \rho\rangle}\left[V_{\mu}\right]\right) .
$$

Démonstration. - On a $q^{\langle\lambda, \rho\rangle}\left[V_{\lambda}\right]=\phi\left(c_{\lambda}\right)+\sum_{\mu<\lambda} P_{\mu, \lambda}(q) \phi\left(c_{\mu}\right)$, où les $P_{\mu, \lambda}$ sont des polynômes de Kazhdan-Lusztig, à coefficients dans $\mathbb{N}$. Cependant le fait que $b_{\lambda}(\mu)$ est entier résulte simplement de la formule (3.7) de [23], avec $w_{0}(\mu)$ à la place de $\mu$ (la formule (3.7) est vraie pour tout $\mu \in X_{*}(T)$ et pas seulement pour $\mu \in \hat{P}^{+}$).

On fixe une clôture algébrique $\overline{\mathbb{Q}}$ de $\mathbb{Q}$. A partir de maintenant le groupe dual $\hat{G}$ est défini sur $\overline{\mathbb{Q}}$.

Pour toute place $\mathfrak{p}$ de $\overline{\mathbb{Q}}$ au-dessus de $p$, on note $v_{\mathfrak{p}}: \overline{\mathbb{Q}} \rightarrow \mathbb{Q} \cup\{+\infty\}$ la valuation $\mathfrak{p}$-adique, normalisée de telle sorte que $v_{\mathfrak{p}}(q)=1, \overline{\mathbb{Q}}_{\mathfrak{p}}$ le complété de $\overline{\mathbb{Q}}$ et $\overline{\mathbb{Z}}_{\mathfrak{p}}=\left\{x \in \overline{\mathbb{Q}}_{\mathfrak{p}}, v_{\mathfrak{p}}(x) \geq 0\right\}$.

On rappelle qu'il y a une bijection naturelle entre l'ensemble des caractères de $\mathcal{H} \otimes_{\mathbb{Z}} \overline{\mathbb{Q}}$ et l'ensemble Conj $^{s s}(\hat{G}(\overline{\mathbb{Q}}))$ des classes de conjugaison semi-simples dans $\hat{G}(\overline{\mathbb{Q}})$ : à une classe de conjugaison $c$ on associe le caractère $\chi$ défini par $\chi\left(\phi^{-1}\left(\left[V_{\lambda}\right]\right)\right)=\operatorname{Tr}\left(c, V_{\lambda}\right)$. Il y a une identification $\operatorname{Conj}^{s s}(\hat{G}(\overline{\mathbb{Q}}))=\hat{T}(\overline{\mathbb{Q}}) / W$. D'autre part la valuation $\mathfrak{p}$-adique $v_{\mathfrak{p}}$ définit un morphisme de groupes $v_{\mathfrak{p}}$ : $\hat{T}(\overline{\mathbb{Q}}) \rightarrow X_{*}(\hat{T}) \otimes_{\mathbb{Z}} \mathbb{R}$, d'où en passant au quotient par $W$, une application

$$
N_{\mathfrak{p}}: \operatorname{Conj}^{s s}(\hat{G}(\overline{\mathbb{Q}}))=\hat{T}(\overline{\mathbb{Q}}) / W \rightarrow\left(X_{*}(\hat{T}) \otimes_{\mathbb{Z}} \mathbb{R}\right) / W=P_{\mathbb{R}}^{+}
$$

que l'on utilisera beaucoup ensuite. On renvoie à [33] pour d'autres considérations sur $N_{\mathfrak{p}}$.

Dans le cas où $G=G L_{r}$ on a $\hat{G}=G L_{r}$ et $N_{\mathfrak{p}}$ est le polygone de Newton $\mathfrak{p}$-adique. En effet

$$
P_{\mathbb{R}}^{+}=\left\{\left(\lambda_{1}, \ldots, \lambda_{r}\right) \in \mathbb{R}^{r}, \lambda_{1} \leq \ldots \leq \lambda_{r}\right\}
$$

et à $\left(\lambda_{1}, \ldots, \lambda_{r}\right) \in P_{\mathbb{R}}^{+}$on associe le polygone convexe dont les sommets sont $\left(i, \lambda_{1}+\ldots+\lambda_{i}\right)$ pour $i \in\{0, \ldots, r\}$. Pour $\lambda=\left(\lambda_{1}, \ldots, \lambda_{r}\right)$ et $\mu=\left(\mu_{1}, \ldots, \mu_{r}\right)$ dans $P_{\mathbb{R}}^{+}, \mu \leq \lambda$ si et seulement si le polygone associé à $\mu$ est au-dessus du polygone associé à $\lambda$ et a les mêmes extrémités. Si $c \in \operatorname{Conj}^{s s}(\hat{G}(\overline{\mathbb{Q}}))$ a pour valeurs propres $\left(\alpha_{1}, \ldots, \alpha_{r}\right)$ ordonnées de telle sorte que $v_{\mathfrak{p}}\left(\alpha_{1}\right) \leq \ldots \leq v_{\mathfrak{p}}\left(\alpha_{r}\right)$ le polygone associé à $\left(v_{\mathfrak{p}}\left(\alpha_{1}\right), \ldots, v_{\mathfrak{p}}\left(\alpha_{r}\right)\right)$ est appelé le polygone de Newton de $c$. 
Corollaire 1.2. - Pour tout caractère $\chi: \mathcal{H} \otimes_{\mathbb{Z}} \overline{\mathbb{Q}} \rightarrow \overline{\mathbb{Q}}$ correspondant à $c \in \operatorname{Conj}^{s s}(\hat{G}(\overline{\mathbb{Q}}))$ et pour toute place $\mathfrak{p}$ de $\overline{\mathbb{Q}}$ au-dessus de $p$, tels que $\chi(\mathcal{H}) \subset$ $\overline{\mathbb{Z}}_{\mathfrak{p}}$, on a $N_{\mathfrak{p}}(c) \leq \rho$ dans $P_{\mathbb{R}}^{+}$.

Démonstration. - D'après la proposition 1.1 l'image de $\mathcal{H}$ par l'isomorphisme de Satake $\&$ est exactement la sous- $\mathbb{Z}$-algèbre de $R(\hat{G}) \otimes_{\mathbb{Z}} \mathbb{Z}\left[q^{\frac{1}{2}}, q^{-\frac{1}{2}}\right]$ ayant pour base sur $\mathbb{Z}$ les $q^{\langle\lambda, \rho\rangle}\left[V_{\lambda}\right]=q^{-\left\langle w_{0}(\lambda), \rho\right\rangle}\left[V_{\lambda}\right]$ pour $\lambda \in \hat{P}^{+}$(on a utilisé $\left.w_{0}(\rho)=-\rho\right)$. On applique alors le lemme suivant en prenant $\nu=\rho$.

Lemme 1.3. - Soit $\nu \in P_{\mathbb{R}}^{+}$et $c \in \operatorname{Conj}^{s s}(\hat{G}(\overline{\mathbb{Q}}))$ tels que

$$
v_{\mathfrak{p}}\left(\operatorname{Tr}\left(c, V_{\lambda}\right)\right) \geq\left\langle w_{0}(\lambda), \nu\right\rangle \text { pour tout } \lambda \in \hat{P}^{+} .
$$

Alors $N_{\mathfrak{p}}(c) \leq \nu$ dans $P_{\mathbb{R}}^{+}$.

Démonstration. - Soit $\lambda \in \hat{P}^{+}$. Soit $r_{\lambda}=\operatorname{dim} V_{\lambda}$. Soit $i \in\left\{1, \ldots, r_{\lambda}\right\}$. Comme tous les poids de $V_{\lambda}$ appartiennent à l'enveloppe convexe des $w(\lambda)$ pour $w \in W$, tous les poids de $\Lambda^{i} V_{\lambda}$ appartiennent à l'enveloppe convexe des $w(i \lambda)$ pour $w \in W$, donc $\Lambda^{i} V_{\lambda}$ se décompose en une somme directe de $V_{\mu}$ avec $\mu \in \hat{P}^{+}$ vérifiant $\mu \leq i \lambda$. On en déduit que

$$
v_{\mathfrak{p}}\left(\operatorname{Tr}\left(c, \Lambda^{i} V_{\lambda}\right)\right) \geq i\left\langle w_{0}(\lambda), \nu\right\rangle .
$$

Soit $\alpha_{1}, \ldots, \alpha_{r_{\lambda}}$ les valeurs propres de $c$ agissant sur $V_{\lambda}$, ordonnées de telle sorte que $v_{\mathfrak{p}}\left(\alpha_{1}\right) \leq \ldots \leq v_{\mathfrak{p}}\left(\alpha_{r_{\lambda}}\right)$. Soit $i \in\left\{1, \ldots, r_{\lambda}\right\}$ tel que $v_{\mathfrak{p}}\left(\alpha_{1}\right)=\ldots=$ $v_{\mathfrak{p}}\left(\alpha_{i}\right)<v_{\mathfrak{p}}\left(\alpha_{i+1}\right)$. Alors $v_{\mathfrak{p}}\left(\operatorname{Tr}\left(c, \Lambda^{i} V_{\lambda}\right)\right)=i v_{\mathfrak{p}}\left(\alpha_{1}\right)$ et on déduit de (4) que $v_{\mathfrak{p}}\left(\alpha_{1}\right) \geq\left\langle w_{0}(\lambda), \nu\right\rangle$. Par ailleurs il est clair que $v_{\mathfrak{p}}\left(\alpha_{1}\right)=\left\langle w_{0}(\lambda), N_{\mathfrak{p}}(c)\right\rangle$ car $w_{0}(\lambda)$ est le plus bas poids de $V_{\lambda}$. On a donc

$$
\left\langle w_{0}(\lambda), N_{\mathfrak{p}}(c)\right\rangle \geq\left\langle w_{0}(\lambda), \nu\right\rangle
$$

pour tout $\lambda \in \hat{P}^{+}$. Comme $\lambda \mapsto-w_{0}(\lambda)$ est une bijection de $\hat{P}^{+}$dans lui-même et comme le cône engendré par les éléments de $\hat{P}^{+}$est le dual du cône engendré par les coracines positives de $\hat{G}$, on déduit de (5) que $N_{\mathfrak{p}}(c) \leq \nu$ dans $P_{\mathbb{R}}^{+}$.

Le corollaire suivant (qui coïncide avec le corollaire 1.2 lorsque $\kappa=0$ ), nous sera utile pour les estimées sur les corps de nombres.

Corollaire 1.4. - Soit $\kappa \in P_{\mathbb{R}}^{+}, \chi: \mathcal{H} \otimes_{\mathbb{Z}} \overline{\mathbb{Q}} \rightarrow \overline{\mathbb{Q}}$ un caractère correspondant à $c \in \operatorname{Conj}^{s s}(\hat{G}(\overline{\mathbb{Q}}))$ et $\mathfrak{p}$ une place de $\overline{\mathbb{Q}}$ au-dessus de $p$, tels que pour tout $\lambda \in \hat{P}^{+}$, on ait $v_{\mathfrak{p}}\left(\chi\left(c_{\lambda}\right)\right) \geq\left\langle w_{0}(\lambda), \kappa\right\rangle$. Alors $N_{\mathfrak{p}}(c) \leq \rho+\kappa$ dans $P_{\mathbb{R}}^{+}$.

Démonstration. - Il résulte de (1) que

$$
q^{\left\langle-w_{0}(\lambda), \rho\right\rangle}\left[V_{\lambda}\right]=\phi\left(c_{\lambda}\right)+\sum_{\mu<\lambda} d_{\lambda}(\mu) \phi\left(c_{\mu}\right)
$$

TOME $139-2011-\mathrm{N}^{\mathrm{O}} 4$ 
avec $d_{\lambda}(\mu) \in \mathbb{Z}$ (égal à $P_{\mu, \lambda}(q)$ dans les notations de la preuve de la proposition 1.1). Pour tout $\lambda \in \hat{P}^{+}$on a donc

$$
v_{\mathfrak{p}}\left(\operatorname{Tr}\left(c, V_{\lambda}\right)\right) \geq\left\langle w_{0}(\lambda), \rho\right\rangle+\min _{\mu \leq \lambda}\left\langle w_{0}(\mu), \kappa\right\rangle=\left\langle w_{0}(\lambda), \rho+\kappa\right\rangle .
$$

On applique alors le lemme 1.3, en prenant $\nu=\rho+\kappa$.

\section{Estimées sur les corps de fonctions}

Soit $F$ le corps des fonctions d'une courbe projective lisse $X$ géométriquement irréductible sur $\mathbb{F}_{q}$. On note $p$ la caractéristique de $\mathbb{F}_{q}$. Soit $\mathbb{A}$ l'anneau des adèles de $F$. Soit $G$ un groupe réductif déployé sur $F$. Soit $K=\prod_{x \in|X|} K_{x}$ un sous-groupe compact de $G(\mathbb{A})$. Pour tout $x \in|X|$ on note $F_{x}$ le complété de $F$ en $x, \vartheta_{F_{x}}$ son anneau d'entiers et $\operatorname{deg}(x)$ le degré de $x$ (de sorte que le corps résiduel de $F_{x}$ est de cardinal $\left.q^{\operatorname{deg}(x)}\right)$. On dit que $x$ est non ramifié si $K_{x}$ est égal à $G\left(\theta_{F_{x}}\right)$ pour un modèle réductif de $G$ sur $\theta_{F_{x}}$, et dans ce cas on note $\mathcal{H}_{x}$ l'algèbre de Hecke associée à $G\left(F_{x}\right)$ et $K_{x}$. Soit $\mathbb{G}_{m}^{s} \subset Z(G)$ la composante connexe du centre de $G$, et $a_{1}, \ldots, a_{s}$ des éléments de $\mathbb{G}_{m}^{s}(\mathbb{A})$ dont les degrés forment un réseau de $\mathbb{Z}^{s}$. On note $\hat{G}$ le groupe dual, défini sur $\overline{\mathbb{Q}}$, et $\hat{T}$ un tore maximal de $\hat{G}$.

Proposition 2.1. - Soit $f: G(F) \backslash G(\mathbb{A}) / K a_{1}^{\mathbb{Z}} \ldots a_{s}^{\mathbb{Z}} \rightarrow \overline{\mathbb{Q}}$ une fonction cuspidale. Soit $x \in|X|$ non ramifié tel que $f$ est propre pour les opérateurs de Hecke en $x$, c'est-à-dire qu'il existe un caractère $\chi: \mathcal{H}_{x} \otimes_{\mathbb{Z}} \overline{\mathbb{Q}} \rightarrow \overline{\mathbb{Q}}$ tel que pour tout $h \in \mathcal{H}_{x}, h . f=\chi(h) f$. Soit $c \in \operatorname{Conj}^{s s}(\hat{G}(\overline{\mathbb{Q}}))$ correspondant à $\chi$. Soit $\mathfrak{p}$ une place de $\overline{\mathbb{Q}}$ au-dessus de $p, v_{\mathfrak{p}}$ la valuation $\mathfrak{p}$-adique sur $\overline{\mathbb{Q}}$, normalisée par $v_{\mathfrak{p}}\left(q^{\operatorname{deg}(x)}\right)=1$ et $N_{\mathfrak{p}}$ comme dans (2). Alors $N_{\mathfrak{p}}(c) \leq \rho$ dans $P_{\mathbb{R}}^{+}$, c'est-à-dire que $N_{\mathfrak{p}}\left(\right.$ c) appartient à l'enveloppe convexe des $w(\rho)$ dans $X_{*}(\hat{T}) \otimes_{\mathbb{Z}} \mathbb{R}$, où $w$ parcourt le groupe de Weyl.

Démonstration. - Grâce au corollaire 1.2, il suffit de montrer que pour tout $h \in \mathcal{H}_{x}, \chi(h)$ appartient à $\overline{\mathbb{Z}}_{\mathfrak{p}}$. Soit $h \in \mathcal{H}_{x}$. Nous allons en fait montrer $\chi(h) \in \overline{\mathbb{Z}}$. On sait qu'il existe une partie finie $D \subset G(F) \backslash G(\mathbb{A}) / K a_{1}^{\mathbb{Z}} \ldots a_{s}^{\mathbb{Z}}$ telle que toute fonction cuspidale soit à support dans $D$. La convolution par $h$ agit sur l'espace des fonctions à support compact sur $G(F) \backslash G(\mathbb{A}) / K a_{1}^{\mathbb{Z}} \ldots a_{s}^{\mathbb{Z}}$ et est donnée par une matrice infinie à coefficients entiers. Soit $M$ la matrice carrée indexée par $D$ extraite de cette matrice infinie. Comme $f$ est à support dans $D, f: D \rightarrow \overline{\mathbb{Q}}$ est un vecteur propre de $M$, de valeur propre $\chi(h)$. D'où $\chi(h) \in \overline{\mathbb{Z}}$.

Soit $\pi$ une représentation automorphe cuspidale pour $G$ à valeurs dans $\overline{\mathbb{Q}}$, dont le caractère central est fini. On suppose que $\pi$ correspond à une représentation $\rho: \pi_{1}(U) \rightarrow \hat{G}\left(\overline{\mathbb{Q}}_{\ell}\right)$, où $U$ est un ouvert de $X$, au sens où $\pi$ est non 
ramifiée en tout point de $U$, et où pour tout $x \in|U|$ la classe de conjugaison semi-simple $c_{x} \in \operatorname{Conj}^{s s}(\hat{G}(\overline{\mathbb{Q}}))$ de $\rho\left(\right.$ Frob $\left._{x}\right)$ (où Frob ${ }_{x}$ est le Frobenius géométrique en $x$ ) est le paramètre de Satake de $\pi$ en $x$ (c'est-à-dire correspond à $\chi$ comme dans la proposition 2.1). La proposition 2.1 montre alors que pour tout $x \in|U|$, pour toute place $\mathfrak{p}$ au-dessus de $p$, si on note $v_{\mathfrak{p}}$ la valuation $\mathfrak{p}$-adique normalisée par $v_{\mathfrak{p}}\left(q^{\operatorname{deg}(x)}\right)=1$, et $N_{\mathfrak{p}}$ comme dans $(2)$, on a $N_{\mathfrak{p}}\left(c_{x}\right) \leq \rho$ dans $P_{\mathbb{R}}^{+}$.

L'estimée de la proposition 2.1 est optimale si on admet la correspondance de Langlands pour $G$. En effet soit $U$ un ouvert de $X$ et $E \rightarrow U$ une courbe elliptique sur $U$ qui n'est pas géométriquement constante. Le composé de la représentation galoisienne associée à $E$, tordue par $\overline{\mathbb{Q}}_{\ell}\left(-\frac{1}{2}\right)$, et du morphisme principal $S L_{2} \rightarrow \hat{G}$ fournit une représentation galoisienne à valeurs dans $\hat{G}\left(\overline{\mathbb{Q}}_{\ell}\right)$, correspondant conjecturalement à une forme automorphe cuspidale, pour laquelle l'estimée ci-dessus est optimale en toute place où $E$ est ordinaire.

Pour $G L_{r}$ la correspondance de Langlands a été montrée par Laurent Lafforgue dans [28], donc on a le corollaire suivant.

Corollaire 2.2. - Soit $U$ un ouvert de $X$, $\ell$ premier à $p$, et $\mathcal{F}$ un faisceau $\ell$-adique lisse irréductible de rang $r$ sur $U$ (à coefficients dans $\overline{\mathbb{Q}}_{\ell}$ ) dont le déterminant est un caractère d'ordre fini. Soit $x \in|U|$. D'après le (i) du théorème VII.6 de [28], les valeurs propres $\alpha_{1}, \ldots, \alpha_{r}$ du Frobenius géométrique Frob $_{x}$ sont dans $\overline{\mathbb{Q}}$. Soit $\mathfrak{p}$ une place de $\overline{\mathbb{Q}}$ au-dessus de $p$ et $v_{\mathfrak{p}}$ la valuation $\mathfrak{p}$-adique, normalisée de telle sorte que $v_{\mathfrak{p}}\left(q^{\operatorname{deg}(x)}\right)=1$. Alors, en permutant les indices pour que $v_{\mathfrak{p}}\left(\alpha_{1}\right) \leq \cdots \leq v_{\mathfrak{p}}\left(\alpha_{r}\right)$, on a pour tout $i \in\{0, \ldots r\}$,

$$
\sum_{j=1}^{i} v_{\mathfrak{p}}\left(\alpha_{j}\right) \geq \sum_{j=1}^{i} \frac{2 j-r-1}{2}=-\frac{i(r-i)}{2}
$$

(ces inégalités sont bien sûr des égalités pour $i=0$ et $i=r$ ).

On peut réexprimer ces inégalités en disant que le polygone de Newton (convexe) des valuations $\mathfrak{p}$-adiques des valeurs propres de Frob ${ }_{x}$, dont les sommets sont les points $\left(i, \sum_{j=1}^{i} v_{\mathfrak{p}}\left(\alpha_{j}\right)\right)$ pour $i=0, \ldots, r$, est au-dessus du polygone convexe de pentes $-\frac{r-1}{2},-\frac{r-3}{2} \ldots, \frac{r-1}{2}$, dont les sommets sont les points $\left(i,-\frac{i(r-i)}{2}\right)$ pour $i=0, \ldots, r$.

Les estimées du corollaire 2.2 impliquent celles proposées par Deligne dans le (iv) de la conjecture (1.2.10) page 155 de [15] et celles montrées par Laurent Lafforgue dans le (iv) du théorème VII.6 de [28].

L'inégalité (6) pour $i=1$ donne $v_{\mathfrak{p}}\left(\alpha_{1}\right) \geq-\frac{r-1}{2}$. Comme $\sum_{j=1}^{r} v_{\mathfrak{p}}\left(\alpha_{j}\right)=0$, L'inégalité (6) pour $i=r-1$ donne $v_{\mathfrak{p}}\left(\alpha_{r}\right) \leq \frac{r-1}{2}$. D'où

$$
v_{\mathfrak{p}}\left(\alpha_{r}\right)-v_{\mathfrak{p}}\left(\alpha_{1}\right) \leq r-1
$$

TOME $139-2011-\mathrm{N}^{\mathrm{O}} 4$ 
qui est exactement l'inégalité figurant page 200 dans la preuve du théorème VII.6 (iv) de [28].

Voici un argument un peu vague qui explique le sens géométrique de l'inégalité (6). Cet argument nécessite beaucoup d'hypothèses supplémentaires. Supposons que $\mathcal{F}$ est à coefficients dans $\mathbb{Q}_{\ell}$ et est géométriquement irréductible (même après avoir étendu ses coefficients à $\overline{\mathbb{Q}}_{\ell}$ ). Supposons que $U$ se relève en une courbe lisse $\tilde{U}$ sur $\vartheta_{F}\left[\frac{1}{n}\right]$, où $F$ est un corps de nombres dont le corps résiduel en une place $v$ est identifié à $\mathbb{F}_{q}, \theta_{F}$ est l'anneau des entiers de $F$ et $n \in \mathbb{N}^{*}$ est premier à $p$ et multiple de $\ell$. Supposons que, pour un certain entier $i \in \mathbb{N}$, le faisceau $\ell$-adique lisse $\mathcal{F}\left(-\frac{i}{2}\right)$ sur $U$ est la restriction d'un faisceau $\ell$-adique lisse $\tilde{\mathcal{F}}$ sur $\tilde{U}$ égal à un groupe de cohomologie en degré $i$ d'une variété projective lisse $\tilde{Y} \rightarrow \tilde{U}$ (le twist à la Tate entre $\mathcal{F}$ et la restriction de $\tilde{\mathcal{F}}$ est nécessaire pour que le déterminant de $\mathcal{F}$ soit d'ordre fini).

Alors les entiers $p$ tels que $h^{p, i-p}$ soit non nul forment un segment dans $\mathbb{Z}$. Autrement à cause de la transversalité de Griffiths un $\mathrm{Fil}^{p}$ non trivial de la cohomologie de de Rham relative de $\tilde{Y} \times_{\text {Spec } \vartheta_{F}\left[\frac{1}{n}\right]}$ Spec $\mathbb{C}$ sur la courbe complexe $\tilde{U} \times_{\operatorname{Spec}} \theta_{F\left[\frac{1}{n}\right]} \operatorname{Spec} \mathbb{C}$ (où l'on a choisi une place à l'infini de $F$ ) serait horizontal pour la connexion de Gauss-Manin, donc la représentation de monodromie de $\pi_{1}\left(\tilde{U} \times_{\operatorname{Spec}} \theta_{F}\left[\frac{1}{n}\right]\right.$ Spec $\left.\mathbb{C}\right)$ sur la cohomologie de Betti relative (tensorisée par $\overline{\mathbb{Q}}$ au-dessus de $\mathbb{Q}$ ) ne serait pas irréductible, donc la représentation de $\pi_{1}^{\text {et,geom }}\left(\tilde{U} \times_{\text {Spec } \theta_{F}\left[\frac{1}{n}\right]} \operatorname{Spec} F\right)$ sur la cohomologie étale relative de $\tilde{Y} \times_{\operatorname{Spec} \theta_{F}\left[\frac{1}{n}\right]}$ Spec $F \operatorname{sur} \tilde{U} \times_{\text {Spec } \theta_{F}\left[\frac{1}{n}\right]} \operatorname{Spec} F$ (tensorisée par $\overline{\mathbb{Q}}_{\ell}$ ) ne serait pas irréductible, et donc $\mathcal{F}$ ne serait pas géométriquement irréductible (même après avoir étendu ses coefficients à $\overline{\mathbb{Q}}_{\ell}$ ).

Puisque les $p$ tels que $h^{p, i-p}$ soit non nul forment un segment dans $\mathbb{Z}$, et que $\frac{\sum_{p} h^{p, i-p} p}{\sum_{p} h^{p, i-p}}=\frac{i}{2}$ (puisque $h^{p, i-p}=h^{i-p, p}$ ), le polygone de Hodge (modifié pour que la pente moyenne soit nulle), plus précisément le polygone convexe dont les pentes sont les $p-\frac{i}{2}$ avec multiplicités $h^{p, i-p}$, est au-dessus du polygone d'extrémités $\left(i,-\frac{i(r-i)}{2}\right)$ pour $i=0, \ldots, r$. D'après les estimées de Katz-Mazur [31, 32] (en supposant vraies les hypothèses techniques sous lesquelles elles sont démontrées) le polygone de Newton (convexe) du Frobenius géométrique pour $\mathcal{F}$ en tout point de $U$ est au-dessus du polygone de Hodge (modifié pour que la pente moyenne soit nulle) donc à fortiori au-dessus du polygone d'extrémités $\left(i,-\frac{i(r-i)}{2}\right)$ pour $i=0, \ldots, r$. On remarquera que lorsque la variété $Y=\tilde{Y} \times_{\tilde{U}} U$ est le $\operatorname{Sym}^{r-1}$ d'une courbe elliptique sur $U$, les trois polygones coïncident aux points de $U$ où la courbe elliptique est ordinaire. 


\section{Estimées sur les corps de nombres}

Pour montrer des estimées sur les valuations $\mathfrak{p}$-adiques des valeurs propres de Hecke de formes automorphes sur les corps de nombres, il faut déjà savoir que ces valeurs propres de Hecke sont des nombres algébriques. Nous nous limiterons aux formes automorphes cohomologiques, c'est-à-dire dont la partie finie apparaît, via la formule de Matsushima, dans la cohomologie de Betti d'un système local sur le quotient d'un espace symétrique par un groupe arithmétique (voir [11] pour une discussion sur l'algébricité des valeurs propres de Hecke). Nous ne considérerons pas les formes automorphes qui apparaissent dans la cohomologie cohérente des fibrés sur les variétés de Shimura (notons simplement que pour les formes modulaires de poids 1, Deligne et Serre montrent directement dans [16] (2.7.3) une estimée pour les valuations $p$-adiques des valeurs propres des opérateurs de Hecke, qui est optimale a posteriori).

Soit $G$ un groupe réductif déployé sur un corps de nombre $F$. On note $B \subset G$ un sous-groupe de Borel, $T \subset B$ un tore maximal non ramifié, et $P^{+} \subset X^{*}(T)$ l'ensemble des poids dominant de $G$.

On note $\vartheta_{F}$ l'anneau des entiers de $F, \mathbb{A}$ les adèles de $F, \mathbb{A}_{f}$ les adèles finies, et $F_{\infty}=F \otimes_{\mathbb{Q}} \mathbb{R}$, de sorte que $\mathbb{A}=\mathbb{A}_{f} \oplus F_{\infty}$. Pour toute place finie $v$ on note $F_{v}$ le complété de $F$ en cette place et $\Theta_{F_{v}}$ son anneau d'entiers. On choisit un plongement de $F$ dans $\overline{\mathbb{Q}}$ et on note $E$ la clôture galoisienne de $F$ dans $\overline{\mathbb{Q}}$ et $\theta_{E}$ son anneau d'entiers. Soit $n=[F: \mathbb{Q}]$ et $\sigma_{1}, \ldots, \sigma_{n}: F \rightarrow E$ les plongements de $F$ dans $E$.

Pour tout $\mu \in P^{+}$, on note $\left(W_{\mu}, \kappa_{\mu}\right)$ la représentation irréductible de $G(E)$ de plus haut poids $\mu$, qui est un $E$-espace vectoriel.

Soient $\mu_{1}, \ldots \mu_{n} \in P^{+}$. On note $(W, \kappa)$ la représentation irréductible $E$ linéaire de $G(F)$ donnée par $W=\bigotimes_{i=1}^{n} W_{\mu_{i}}$ et $\kappa=\bigotimes_{i=1}^{n} \kappa_{\mu_{i}} \circ \sigma_{i}$ (en notant $\sigma_{i}: G(F) \rightarrow G(E)$ l'inclusion induite par $\left.\sigma_{i}\right)$.

Soit $K_{\infty}$ un compact maximal de $G\left(F_{\infty}\right)$. Pour tout sous-groupe ouvert compact $K \operatorname{de} G\left(\mathbb{A}_{f}\right)$ on note

$$
S_{K}=G(F) \backslash G(\mathbb{A}) / K K_{\infty} .
$$

Soit $K=\prod_{v} K_{v}$ un sous-groupe compact ouvert de $G\left(\mathbb{A}_{f}\right)$, où le produit a lieu sur les places finies de $F$. On suppose $K$ assez petit pour que $G(F)$ agisse librement sur $G(\mathbb{A}) / K K_{\infty}$.

On note $W$ le système local en $E$-espaces vectoriels $W \times_{G(F)} G(\mathbb{A}) / K K_{\infty}$ sur $S_{K}$, c'est-à-dire le quotient de $W \times G(\mathbb{A}) / K K_{\infty}$ par l'action de $G(F)$ donnée par $h \mapsto((x, g) \mapsto(\kappa(h) x, h g))$, pour $h \in G(F), x \in W$ et $g \in G(\mathbb{A}) / K K_{\infty}$.

Pour tout $k \in \mathbb{N}, H^{k}\left(S_{K}, W\right)$ est un $E$-espace vectoriel de dimension finie. En effet il existe un ensemble fini $\mathcal{f}$ et des sous-groupes discrets sans torsion 
$\Gamma_{j} \subset G(F)$ pour $j \in \mathcal{J}$ tels qu'on ait une égalité $G(F)$-équivariante

$$
G\left(\mathbb{A}_{f}\right) / K=\bigcup_{j \in \mathcal{J}} G(F) / \Gamma_{j}
$$

Il en résulte

$$
S_{K}=\bigcup_{j \in \mathcal{J}} \Gamma_{j} \backslash G\left(F_{\infty}\right) / K_{\infty}
$$

La restriction de $W$ à $\Gamma_{j} \backslash G\left(F_{\infty}\right) / K_{\infty}$ s'identifie à $W \times_{\Gamma_{j}} G\left(F_{\infty}\right) / K_{\infty}$. Enfin $H^{k}\left(\Gamma_{j} \backslash G\left(F_{\infty}\right) / K_{\infty}, W\right)$ est de dimension finie car $\Gamma_{j} \backslash G\left(F_{\infty}\right) / K_{\infty}$ est muni d'une action libre de $\left(\mathbb{R}_{+}^{*}\right)^{z}$ (où $z$ est la dimension sur $F$ du centre de $G$ ), de telle sorte que le quotient soit l'intérieur d'une variété à coins compacte (la compactification de Borel-Serre, voir [7] et [6] III.5).

On suppose $G$ muni d'un modèle réductif sur $\theta_{F}$ tel que $K_{v} \subset G\left(\theta_{F_{v}}\right)$ pour toute place finie $v$ de $F$. Soit $v$ une place finie de $F$ telle que $K_{v}=G\left(\theta_{F_{v}}\right.$ ) (on dira que $v$ est non ramifiée). Soit $q_{v}$ le cardinal du corps résiduel de $F_{v}, p_{v}$ sa caractéristique et $\pi_{v}$ une uniformisante de $F_{v}$. On note $\mathcal{H}_{v}$ l'algèbre de Hecke associée à $G\left(F_{v}\right)$ et à $G\left(\theta_{F_{v}}\right)$.

On note $\hat{G}$ le groupe dual de $G$, défini sur $\overline{\mathbb{Q}}$, et $\hat{T}$ un tore maximal de $\hat{G}$.

Proposition 3.1. - Soit $v$ une place finie de $F$ telle que $K_{v}=G\left(\theta_{F_{v}}\right)$. Soit

$$
f \in H^{\bullet}\left(S_{K}, W\right) \otimes_{E} \overline{\mathbb{Q}}
$$

propre par les opérateurs de Hecke en $v$, selon un caractère $\chi: \mathscr{H}_{v} \otimes_{\mathbb{Z}} \overline{\mathbb{Q}} \rightarrow \overline{\mathbb{Q}}$. Soit $c \in \operatorname{Conj}^{s s}(\hat{G}(\overline{\mathbb{Q}}))$ correspondant à $\chi$. Alors pour toute place $\mathfrak{p}$ de $\overline{\mathbb{Q}}$ audessus de $p_{v}$, si la valuation $\mathfrak{p}$-adique est normalisée par $v_{\mathfrak{p}}\left(q_{v}\right)=1$, et $N_{\mathfrak{p}}$ comme dans (2),

$$
N_{\mathfrak{p}}(c) \leq \rho+\mu \quad \text { dans } \quad P_{\mathbb{R}}^{+} \quad \text { où } \quad \mu=\frac{1}{\left[F_{v}: \mathbb{Q}_{p_{v}}\right]} \sum_{i \text { tel que } \mathfrak{p} \text { divise } \sigma_{i}(v)} \mu_{i}
$$

c'est-à-dire que $N_{\mathfrak{p}}(c)$ appartient à l'enveloppe convexe des $w(\rho+\mu)$ dans $X_{*}(\hat{T}) \otimes_{\mathbb{Z}} \mathbb{R}$, où $w$ parcourt le groupe de Weyl.

Remarque. - Le nombre de $i$ tels que $\mathfrak{p}$ divise l'image de $v$ par $\sigma_{i}$ est exactement $\left[F_{v}: \mathbb{Q}_{p_{v}}\right]$. Donc la somme qui donne $\mu$ est une moyenne.

Démonstration de la proposition 3.1. - En suivant Langlands [29], Harder, Clozel [12] et Mauger [30], on va construire un système local en $\theta_{E} \otimes_{\mathbb{Z}} \mathbb{Z}_{p_{v}}{ }^{-}$ modules libres $W_{\mathbb{Z}_{p_{v}}}$ sur $S_{K}$ muni d'un isomorphisme de systèmes locaux en $E \otimes_{\mathbb{Q}} \mathbb{Q}_{p_{v}}$-modules libres

$$
W_{\mathbb{Z}_{p_{v}}} \otimes_{\vartheta_{E} \otimes_{\mathbb{Z}} \mathbb{Z}_{p_{v}}}\left(E \otimes_{\mathbb{Q}} \mathbb{Q}_{p_{v}}\right)=W_{\mathbb{Q}_{p_{v}}}
$$


où l'on note $\mathcal{W}_{\mathbb{Q}_{p_{v}}}=W \otimes_{\mathbb{Q}} \mathbb{Q}_{p_{v}}$. On rappelle que $E \otimes_{\mathbb{Q}} \mathbb{Q}_{p_{v}}$ est le produit des complétés de $E$ en les places au-dessus de $p_{v}$ et que $\theta_{E} \otimes_{\mathbb{Z}} \mathbb{Z}_{p_{v}}$ est le produit de leurs anneaux d'entiers.

Pour tout $i \in\{1, \ldots, n\}$, la représentation $\kappa_{\mu_{i}}$ s'étend en une représentation continue de $G\left(E \otimes_{\mathbb{Q}} \mathbb{Q}_{p_{v}}\right)$ sur le $E \otimes_{\mathbb{Q}} \mathbb{Q}_{p_{v}}$-module $W_{\mu_{i}} \otimes_{\mathbb{Q}} \mathbb{Q}_{p_{v}}$. Or $G\left(\theta_{E} \otimes_{\mathbb{Z}} \mathbb{Z}_{p_{v}}\right)$ est un schéma en groupes de Chevalley (voir [10,2] et les chapitres XXII à XXV de [17]). D'après [10, 36, 2, 27] il existe un $\Theta_{E} \otimes_{\mathbb{Z}} \mathbb{Z}_{p_{v}}$-module libre $W_{\mu_{i}, \mathbb{Z}_{p_{v}}}$ qui est un réseau dans $W_{\mu_{i}} \otimes_{\mathbb{Q}} \mathbb{Q}_{p_{v}}$, qui est préservé par $G\left(\theta_{E} \otimes_{\mathbb{Z}} \mathbb{Z}_{p_{v}}\right)$ et admet une décomposition par les poids. Il en résulte que pour tout $\lambda \in \hat{P}^{+}$on a

$$
\kappa_{\mu_{i}} \circ \sigma_{i}\left(\lambda\left(\pi_{v}\right)\right)\left(W_{\mu_{i}, \mathbb{Z}_{p_{v}}}\right) \subset \sigma_{i}\left(\pi_{v}\right)^{\left\langle w_{0}(\lambda), \mu_{i}\right\rangle} W_{\mu_{i}, \mathbb{Z}_{p_{v}}}
$$

où $\pi_{v}$ désigne ici l'élément de $\vartheta_{F} \otimes_{\mathbb{Z}} \mathbb{Z}_{p_{v}}=\oplus_{u \mid p_{v}} \vartheta_{F_{u}}$ égal à $\pi_{v}$ pour $u=v$ et à 1 pour $u \neq v$, et où $\sigma_{i}: \theta_{F} \otimes_{\mathbb{Z}} \mathbb{Z}_{p_{v}} \rightarrow \theta_{E} \otimes_{\mathbb{Z}} \mathbb{Z}_{p_{v}}$ désigne l'extension par continuité de $\sigma_{i}: \theta_{F} \rightarrow \theta_{E}$.

Le $\vartheta_{E} \otimes_{\mathbb{Z}} \mathbb{Z}_{p_{v}}$-module libre $W_{\mathbb{Z}_{p_{v}}}=\bigotimes_{i=1}^{n} W_{\mu_{i}, \mathbb{Z}_{p_{v}}}$ est un réseau dans $W \otimes_{\mathbb{Q}}$ $\mathbb{Q}_{p_{v}}$ et on le munit de la représentation $\kappa=\bigotimes_{i=1}^{n} \kappa_{\mu_{i}} \circ \sigma_{i}$ de $G\left(\Theta_{F} \bigotimes_{\mathbb{Z}} \mathbb{Z}_{p_{v}}\right)$. Pour tout $\lambda \in \hat{P}^{+}$on a

$$
\kappa\left(\lambda\left(\pi_{v}\right)\right)\left(W_{\mathbb{Z}_{p_{v}}}\right) \subset \prod_{i=1}^{n} \sigma_{i}\left(\pi_{v}\right)^{\left\langle w_{0}(\lambda), \mu_{i}\right\rangle} W_{\mathbb{Z}_{p_{v}}} .
$$

On pose alors

$$
W_{\mathbb{Z}_{p_{v}}}=\left(G(F) \backslash G(\mathbb{A}) / K_{\infty} \prod_{u \nmid p_{v}} K_{u}\right) \times \prod_{u p_{v}} K_{u} W_{\mathbb{Z}_{p_{v}}}
$$

où $\prod_{u \mid p_{v}} K_{u} \subset \prod_{u \mid p_{v}} G\left(\vartheta_{F_{u}}\right)=G\left(\vartheta_{F} \otimes_{\mathbb{Z}} \mathbb{Z}_{p_{v}}\right)$ agit sur $W_{\mathbb{Z}_{p_{v}}}$ par $\kappa$. Alors (9) vient de l'isomorphisme

$$
\begin{gathered}
\left(W \otimes_{\mathbb{Q}} \mathbb{Q}_{p_{v}}\right) \times_{G(F)} G(\mathbb{A}) / K K_{\infty} \\
=\left(G(F) \backslash G(\mathbb{A}) / K_{\infty} \prod_{u \nmid p_{v}} K_{u}\right) \times \prod_{u \mid p_{v}} K_{u}\left(W \otimes_{\mathbb{Q}} \mathbb{Q}_{p_{v}}\right)
\end{gathered}
$$

qui, pour $x \in W \otimes_{\mathbb{Q}} \mathbb{Q}_{p_{v}}$ et $g \in G(\mathbb{A})$ envoie $(x, g)$ sur $\left(g, \kappa\left(g_{p_{v}}^{-1}\right) x\right)$ où $g_{p_{v}}$ désigne la composante de $g$ dans $\prod_{u \mid p_{v}} G\left(F_{u}\right)$ et où $\kappa$ désigne ici la représentation de $\prod_{u \mid p_{v}} G\left(F_{u}\right)=G\left(F \otimes_{\mathbb{Q}} \mathbb{Q}_{p_{v}}\right)$ sur $W \otimes_{\mathbb{Q}} \mathbb{Q}_{p_{v}}$.

Soit $\bar{H}^{\bullet}\left(S_{K}, W_{\mathbb{Z}_{p_{v}}}\right)$ l'image de $H^{\bullet}\left(S_{K}, W_{\mathbb{Z}_{p_{v}}}\right)$ dans

$$
H^{\bullet}\left(S_{K}, W_{\mathbb{Q}_{p_{v}}}\right)=H^{\bullet}\left(S_{K}, W\right) \otimes_{\mathbb{Q}} \mathbb{Q}_{p_{v}}
$$

par l'identification (9). C'est un $\vartheta_{E} \otimes_{\mathbb{Z}} \mathbb{Z}_{p_{v}}$-module libre et un réseau de (13) pour la raison suivante. Pour $j \in \mathcal{J}$ soit $y_{j} \in G\left(\mathbb{A}_{f}\right)$ tel que $y_{j} K \in$ $G\left(\mathbb{A}_{f}\right) / K$ corresponde à $1 \in G(F) / \Gamma_{j}$ par (7). Alors l'image de $\Gamma_{j}$ par $G(F) \rightarrow$ $G\left(\mathbb{A}_{f}\right) \stackrel{\gamma \mapsto y_{j}^{-1} \gamma y_{j}}{\longrightarrow} G\left(\mathbb{A}_{f}\right)$ est incluse dans $K$ et on note $\alpha_{j, p_{v}}: \Gamma_{j} \rightarrow \prod_{u \mid p_{v}} K_{u}$ 
le morphisme qui s'en déduit. Alors la restriction de $W_{\mathbb{Z}_{p_{v}}}$ à $\Gamma_{j} \backslash G\left(F_{\infty}\right) / K_{\infty}$ par (8) s'identifie à $W_{\mathbb{Z}_{p_{v}}} \times_{\Gamma_{j}} G\left(F_{\infty}\right) / K_{\infty}$ où $\Gamma_{j}$ agit sur $W_{\mathbb{Z}_{p_{v}}}$ par $\kappa \circ \alpha_{j, p_{v}}$. On reprend alors l'argument de la compactification de Borel-Serre. La notation $\bar{H}^{\bullet}\left(S_{K}, W_{\mathbb{Z}_{p_{v}}}\right)$ est justifiée par le fait que ce $\theta_{E} \otimes_{\mathbb{Z}} \mathbb{Z}_{p_{v}}$-module libre est le quotient de $H^{\bullet}\left(S_{K}, W_{\mathbb{Z}_{p_{v}}}\right)$ par sa partie de torsion.

D'après [12], pour tout $\lambda \in \hat{P}^{+}$l'action de $c_{\lambda} \in \mathcal{H}_{v}$ par convolution à droite sur (13) est la suivante. On considère le diagramme

$$
\begin{aligned}
& S_{\prod_{u \neq v} K_{u}\left(K_{v} \cap \lambda\left(\pi_{v}\right) K_{v} \lambda\left(\pi_{v}\right)^{-1}\right)} \stackrel{m_{\lambda\left(\pi_{v}\right)}}{\longrightarrow} S_{\prod_{u \neq v} K_{u}\left(\lambda\left(\pi_{v}\right)^{-1} K_{v} \lambda\left(\pi_{v}\right) \cap K_{v}\right)} \\
& \downarrow p_{1} \quad \downarrow p_{2} \\
& S_{K} \quad S_{K}
\end{aligned}
$$

où $m_{\lambda\left(\pi_{v}\right)}$ est $g \mapsto g \lambda\left(\pi_{v}\right)$. L'action de $c_{\lambda}$ sur (13) est la composée

- de l'image inverse par $p_{2} m_{\lambda\left(\pi_{v}\right)}$,

- du morphisme de systèmes locaux sur $S_{\prod_{u \neq v} K_{u}\left(K_{v} \cap \lambda\left(\pi_{v}\right) K_{v} \lambda\left(\pi_{v}\right)^{-1}\right)}$

$$
\left(p_{2} m_{\lambda\left(\pi_{v}\right)}\right)^{*}\left(W_{\mathbb{Q}_{p_{v}}}\right) \rightarrow p_{1}^{*}\left(\mathcal{W}_{\mathbb{Q}_{p_{v}}}\right)
$$

qui est tautologique si on décrit $W_{\mathbb{Q}_{p_{v}}}$ par (11) et qui est donné par $\kappa\left(\lambda\left(\pi_{v}\right)\right)$ si on décrit $W_{\mathbb{Q}_{p_{v}}}$ par (12),

- et de la trace par $p_{1}$.

Il résulte de (10) que pour tout $\lambda \in \hat{P}^{+}$l'action de $c_{\lambda}$ par convolution sur (13) vérifie

$$
c_{\lambda} \cdot \bar{H}^{\bullet}\left(S_{K}, W_{\mathbb{Z}_{p_{v}}}\right) \subset \prod_{i=1}^{n} \sigma_{i}\left(\pi_{v}\right)^{\left\langle w_{0}(\lambda), \mu_{i}\right\rangle} \bar{H}^{\bullet}\left(S_{K}, W_{\mathbb{Z}_{p_{v}}}\right)
$$

Comme la valuation $\mathfrak{p}$-adique $v_{\mathfrak{p}}$ est normalisée par $v_{\mathfrak{p}}\left(q_{v}\right)=1, v_{\mathfrak{p}}\left(\sigma_{i}\left(\pi_{v}\right)\right)$ est égal à $\frac{1}{\left[F_{v}: \mathbb{Q}_{p_{v}}\right]}$ si $\mathfrak{p}$ divise l'image de $v$ par $\sigma_{i}$ (qui est une place de $\sigma_{i}(F) \subset \overline{\mathbb{Q}}$ ) et vaut 0 sinon. Grâce au corollaire 1.4, l'inclusion (15) implique la proposition.

REMARQUe. - Pour tout nombre premier $p$ on peut introduire un système local en $\theta_{E} \otimes_{\mathbb{Z}} \mathbb{Z}_{p}$-modules libres $W_{\mathbb{Z}_{p}}$ sur $S_{K}$ qui est un réseau dans $W \otimes_{\mathbb{Q}} \mathbb{Q}_{p}$, comme on l'a fait pour $p=p_{v}$. Cela permet de définir un système local $W_{\mathbb{Z}}$ en $\theta_{E}$-modules projectifs de type fini (pas nécessairement libres) sur $S_{K}$ tel que $W_{\mathbb{Z}} \otimes_{\mathbb{Z}} \mathbb{Q}=W$ et $W_{\mathbb{Z}} \otimes_{\mathbb{Z}} \mathbb{Z}_{p}=W_{\mathbb{Z}_{p}}$ pour tout $p$. En effet on pose $W_{\mathbb{Z}}=$ $W \cap \bigcap_{p} W_{\mathbb{Z}_{p}}$ où $p$ parcourt les nombres premiers. Le système local $W_{\mathbb{Z}}$ apparaît dans [12].

La proposition 3.1 implique des estimées pour les représentations automorphes cuspidales cohomologiques. 
D'après [3, 4, 5] et plus spécialement le corollaire 5.5 de [4], si $G$ est semisimple, toutes les représentations automorphes cuspidales dont la composante à l'infini est cohomologique apparaissent dans

$$
\varliminf_{\longrightarrow} H^{\bullet}\left(S_{K}, W\right) \otimes_{E} \overline{\mathbb{Q}}
$$

(où la limite est prise sur $K$ de plus en plus petit) pour certains $\mu_{1}, \ldots \mu_{n}$. Cela est expliqué aussi dans les paragraphes 4.1 et 4.2 de [35]. Pour $G=G L_{n}$ cela figure dans le lemme 3.15 de [11].

Notons que les caractères infinitésimaux $\nu_{i}$ des composantes à l'infini d'une représentation automorphe cuspidale $\pi$ dont la partie finie apparaît (par la formule de Matsushima) dans (16) sont les $\rho-w_{0}\left(\mu_{i}\right)=-w_{0}\left(\rho+\mu_{i}\right)$. Pour des rappels sur les caractères infinitésimaux on renvoie à [37]. Pour $G=G L_{n}$ cela figure dans le paragraphe 3.5 de [11]. Pour $G$ semi-simple on peut le déduire des théorèmes 5.5 et 5.6 et de la proposition 6.4 de [38]. Ceci permet de réécrire la proposition 3.1 en termes des caractères infinitésimaux $\nu_{i}$ des composantes à l'infini : on a

$$
\rho+\mu=-w_{0}\left(\frac{1}{\left[F_{v}: \mathbb{Q}_{p_{v}}\right]} \sum_{i \text { tel que } \mathfrak{p} \text { divise } \sigma_{i}(v)} \nu_{i}\right)
$$

puisque, d'après la remarque qui suit la proposition 3.1, la somme qui donne $\mu$ est une moyenne.

\section{Estimées pour les valuations $p$-adiques des valeurs propres de Frobenius pour les motifs}

On déduit facilement d'un résultat de Katz-Mazur [31, 32] que si l'on considère la cohomologie d'une variété projective lisse sur un corps de nombres, en presque toutes les places le polygone de Newton (convexe) des valuations $p$-adiques du Frobenius géométrique est au-dessus du polygone de Hodge. La proposition 4.1 ci-dessous établit ces estimées en toutes les places non ramifiées pour les motifs sur un corps de nombres à coefficients dans $\overline{\mathbb{Q}}$. La démonstration utilise seulement la réalisation cristalline du motif, et le fait que la condition de faible admissibilité en théorie de Fontaine implique elle aussi que le polygone de Newton est au-dessus du polygone de Hodge, comme Fontaine l'a montré dans la proposition 4.3.3. de [18] (voir aussi la remarque 4.4.6 de [21]). La référence à la proposition 4.3.3. de [18] figure explicitement dans [12] et [25]. Ce paragraphe ne contient donc rien d'original.

A la fin de ce paragraphe nous vérifierons que, par la correspondance conjecturale entre motifs simples et représentations automorphes cuspidales algébriques pour $G=G L_{r}$, les estimées des propositions 4.1 et 3.1 sont absolument identiques. Remarquons néanmoins que la proposition 3.1 ne s'applique qu'aux

TOME $139-2011-\mathrm{N}^{\mathrm{O}} 4$ 
formes automorphes cuspidales cohomologiques, alors que la proposition 4.1 s'applique à tous les motifs.

On note $\hat{T}$ le tore maximal de $G L_{r}$ formé des matrices diagonales (pour les estimées qui vont suivre $G L_{r}$ joue le même rôle que $\hat{G}$ dans le paragraphe précédent).

Soit $E$ et $F$ des corps de nombres tels que $E$ contienne la clôture galoisienne de $F$. On fixe aussi un plongement $E \subset \overline{\mathbb{Q}}$. Soient $n=[F: \mathbb{Q}]$ et $\sigma_{1}, \ldots, \sigma_{n}$ les plongements de $F$ dans $E$. Pour toute place finie $v$ de $F$, on note $F_{v}$ le complété de $F$ en $v, k_{F_{v}}$ son corps résiduel, $q_{v}$ le cardinal de $k_{F_{v}}$ et $p_{v}$ sa caractéristique. Pour toute place finie $w$ de $E$, on note $E_{w}$ le complété de $E$ en $w$.

Soit $M$ un motif de rang $r$ sur $F$, à coefficients dans $E$. On renvoie à [19, 22] et [37] pour les différentes réalisations de $M$ et les compatibilités entre elles. On utilisera ici

- la réalisation de de Rham $M_{\mathrm{d} R}$ qui est un module libre de rang $r$ sur $F \otimes_{\mathbb{Q}} E$ muni d'une filtration,

- pour toute place finie $w$ de $E$ la réalisation $w$-adique $M_{w}$ qui est un $E_{w}$-espace vectoriel de rang $r$ muni d'une représentation continue de $\operatorname{Gal}(\bar{F} / F)$,

- et pour toute place finie $v$ de $F$ une représentation $W D_{v}(M)$ de rang $r$ du groupe de Weil-Deligne $W D\left(F_{v}\right)$, qui est rationnelle sur $E$ et définie $\operatorname{sur} \overline{\mathbb{Q}}$,

en imposant les compatibilités suivantes :

- pour toute place finie $v$ de $F$ on a un isomorphisme $\operatorname{Gal}\left(\bar{F}_{v} / F_{v}\right)$ équivariant de $B_{\mathrm{d} R} \otimes_{\mathbb{Q}} E$-modules filtrés

$$
B_{\mathrm{d} R} \otimes_{\mathbb{Q}_{p_{v}}}\left(\bigoplus_{w \mid p_{v}} M_{w}\right) \simeq B_{\mathrm{d} R} \otimes_{F} M_{\mathrm{d} R}
$$

où la somme porte sur les places $w$ de $E$ au-dessus de $p_{v}$,

- pour toute place finie $v$ de $F$, pour toute place $w$ de $E$ au-dessus de $p_{v}$ et pour toute place $\mathfrak{p}$ de $\overline{\mathbb{Q}}$ au-dessus de $w$, l'extension des scalaires de $\overline{\mathbb{Q}}$ au complété $\overline{\mathbb{Q}}_{\mathfrak{p}}$ de $\overline{\mathbb{Q}}$ en $\mathfrak{p}$ de $W D_{v}(M)$ est la représentation de Weil-Deligne associée à la représentation potentiellement semi-stable $\left.M_{w}\right|_{\mathrm{Gal}\left(\bar{F}_{v} / F_{v}\right)}$ (comme dans [20]).

On dit que $M$ est non ramifié en $v$ si $W D_{v}(M)$ est non ramifiée. Dans ce cas le Frobenius géométrique en $v$ détermine $c_{v} \in \operatorname{Conj}^{s s}\left(G L_{r}(\overline{\mathbb{Q}})\right)$. Soit $\mathfrak{p}$ une place de $\overline{\mathbb{Q}}$ au-dessus de $p_{v}, v_{\mathfrak{p}}$ la valuation $\mathfrak{p}$-adique, normalisée par $v_{\mathfrak{p}}\left(q_{v}\right)=1$ et $N_{\mathfrak{p}}$ comme dans (2).

Pour tout $i \in\{1, \ldots, n\}$, soit $\nu_{i}=\left(\nu_{i}^{1}, \ldots, \nu_{i}^{r}\right) \in X_{*}(\hat{T})=\mathbb{Z}^{r}$ (avec $\nu_{i}^{1} \leq$ $\cdots \leq \nu_{i}^{r}$ ) le $r$-uplet des pentes de Hodge associé à $\sigma_{i}$. Plus précisément on a un isomorphisme $F \otimes_{\mathbb{Q}} E=\bigoplus_{i=1}^{n} E$ qui envoie $x \otimes y \operatorname{sur}\left(\sigma_{i}(x) y\right)_{i \in\{1, \ldots, n\}}$, d'où une 
décomposition $M_{d R}=\bigoplus_{i=1}^{n} M_{d R, i}$, où $M_{d R, i}$ est un $E$-espace vectoriel filtré de rang $r$, dont $\left(\nu_{i}^{1}, \ldots, \nu_{i}^{r}\right)$ est le $r$-uplet des pentes de Hodge, c'est-à-dire, en notant ... $\mathrm{Fil}_{i}^{k} \supset \mathrm{Fil}_{i}^{k+1} \ldots$ la filtration de $M_{d R, i}, \operatorname{dim} \mathrm{Fil}_{i}^{k}=\sharp\left\{j, \nu_{i}^{j} \geq k\right\}$.

Proposition 4.1. - Pour toute place $v$ de $F$ où $M$ est non ramifié, pour toute place $\mathfrak{p}$ de $\overline{\mathbb{Q}}$ au-dessus de $p_{v}$,

$$
N_{\mathfrak{p}}\left(c_{v}\right) \leq \nu \text { où } \nu=\frac{1}{\left[F_{v}: \mathbb{Q}_{p_{v}}\right]} \sum_{i \text { tel que } \mathfrak{p} \text { divise } \sigma_{i}(v)} \nu_{i}
$$

autrement dit le polygone de Newton p-adique normalisé du Frobenius géométrique en $v$ est au-dessus du polygone (convexe) associé à $\nu$ qui est la moyenne des $r$-uplets de Hodge correspondant aux plongements $\sigma_{i}: F \rightarrow \overline{\mathbb{Q}}$ tels que $\mathfrak{p}$ soit au-dessus de $\sigma_{i}(v)$.

Démonstration. - Soit $v$ une place de $F$ où $M$ est non ramifié. On note $F_{v, 0}=$ $W\left(k_{F_{v}}\right)\left[\frac{1}{p_{v}}\right]$ la plus grande extension non ramifiée de $\mathbb{Q}_{p_{v}}$ incluse dans $F_{v}$. La réalisation cristalline de $M$ en $v$ est définie par

$$
M_{\text {cris }, v}=\left(B_{\text {cris }} \otimes_{\mathbb{Q}_{p_{v}}}\left(\bigoplus_{w \mid p_{v}} M_{w}\right)\right)^{\operatorname{Gal}\left(\bar{F}_{v} / F_{v}\right)} .
$$

C'est un module libre de rang $r$ sur $F_{v, 0} \otimes_{\mathbb{Q}} E$ muni d'un automorphisme $\operatorname{Fr} \otimes 1$ linéaire $\varphi_{v}$, où Fr est l'automorphisme de $F_{v, 0}$ qui induit $x \mapsto x^{p_{v}}$ sur le corps résiduel.

On a $\mathbb{Q}_{p_{v}} \otimes_{\mathbb{Q}} E=\bigoplus_{w \mid p_{v}} E_{w}$, d'où une décomposition

$$
M_{\mathrm{cris}, v}=\bigoplus_{w \mid p_{v}} M_{\mathrm{cris}, v, w}
$$

où $M_{\text {cris }, v, w}$ est un $F_{v, 0} \otimes_{\mathbb{Q}_{p_{v}}} E_{w}$-module libre de rang $r$ muni d'un automorphisme $\operatorname{Fr} \otimes 1$-linéaire $\varphi_{v}$.

Le lemme suivant, appliqué à $D=M_{\text {cris }, v, w}$, montre que pour toute place $w$ de $E$ au-dessus de $p_{v}$, et pour tout place $\mathfrak{p}$ de $\overline{\mathbb{Q}}$ au-dessus de $w$, le polygone de Newton (convexe) p-adique normalisé du Frobenius géométrique en $v$ est au-dessus du polygone (convexe) associé à la moyenne des $r$-uplets de Hodge correspondant aux plongements $\sigma: F_{v} \rightarrow E_{w}$. On renvoie à [20] et au premier paragraphe de [37] pour la règle qui permet d'associer une représentation de $W D\left(F_{v}\right)$ à un $\left(\varphi, N, \operatorname{Gal}\left(L / F_{v}\right)\right)$-module filtré (ici $L=F_{v}$ et $N=0$ puisque qu'on suppose $M$ non ramifié en $v$ ). Ceci termine la preuve de la proposition 4.1.

Soient $v, F_{v}, F_{v, 0}, q_{v}, p_{v}$, Fr comme ci-dessus et $s$ l'entier tel que $q_{v}=p_{v}^{s}$. Soit $w$ une place finie de $E$ au-dessus de $p_{v}$ et $E_{w}$ comme ci-dessus. Soit $\mathfrak{p}$ 
une place de $\overline{\mathbb{Q}}$ au-dessus de $w$. On note $v_{\mathfrak{p}}$ la valuation sur $\overline{\mathbb{Q}}$ normalisée par $v_{\mathfrak{p}}\left(q_{v}\right)=1$ et

$$
N_{\mathfrak{p}}: \operatorname{Conj}^{s s}\left(\hat{G}\left(\overline{\mathbb{Q}}_{\mathfrak{p}}\right)\right) \rightarrow P_{\mathbb{R}}^{+}=\left\{\left(\lambda_{1}, \ldots, \lambda_{r}\right) \in \mathbb{R}^{r}, \lambda_{1} \leq \ldots \leq \lambda_{r}\right\}
$$

l'application qui à une classe de conjugaison $c$ de valeurs propres $\left(\alpha_{1}, \ldots, \alpha_{r}\right)$ ordonnées de telle sorte que $v_{\mathfrak{p}}\left(\alpha_{1}\right) \leq \ldots \leq v_{\mathfrak{p}}\left(\alpha_{r}\right)$ associe $\left(v_{\mathfrak{p}}\left(\alpha_{1}\right), \ldots, v_{\mathfrak{p}}\left(\alpha_{r}\right)\right)$. Comme $E_{w}$ contient la clôture galoisienne de $F_{v}$, on a un isomorphisme

$$
F_{v} \otimes_{\mathbb{Q}_{p}} E_{w}=\bigoplus_{\sigma} E_{w}
$$

qui à $x \otimes y$ associe $(\sigma(x) y)_{\sigma}$, où $\sigma$ parcourt les plongements de $F_{v}$ dans $E_{w}$. De même on a un isomorphisme

$$
F_{v, 0} \otimes_{\mathbb{Q}_{p_{v}}} E_{w}=\bigoplus_{\tau} E_{w}
$$

qui à $x \otimes y$ associe $(\tau(x) y)_{\tau}$, où $\tau$ parcourt les plongements de $F_{v, 0}$ dans $E_{w}$.

Lemme 4.2. - Soit $D$ un $F_{v, 0} \otimes_{\mathbb{Q}_{p_{v}}} E_{w}$-module libre de rang $r$, muni d'un automorphisme $\operatorname{Fr} \otimes 1$-linéaire $\varphi$. Soit $\left(\mathrm{Fil}^{i} D_{F_{v}}\right)_{i \in \mathbb{Z}}$ une filtration de

$$
D_{F_{v}}:=D \otimes F_{v, 0} F_{v}
$$

par des sous- $F_{v} \otimes_{\mathbb{Q}_{p_{v}}} E_{w}$-modules (non nécessairement libres). On suppose que le $\varphi$-module filtré $D$ est faiblement admissible, c'est-à-dire que $t_{H}(D)=t_{N}(D)$ et que pour tout sous- $F_{v, 0} \otimes_{\mathbb{Q}_{p_{v}}} E_{w}$-module $D^{\prime}$ de $D$ stable par $\varphi$ (et donc libre), on a $t_{H}\left(D^{\prime}\right) \leq t_{N}\left(D^{\prime}\right)$. On rappelle que

$$
t_{N}\left(D^{\prime}\right)=v_{\mathfrak{p}}\left(\operatorname{det}_{E_{w}}\left(\left.\varphi\right|_{D^{\prime}}\right)\right)
$$

(où $\operatorname{det}_{E_{w}}\left(\left.\varphi\right|_{D^{\prime}}\right)$ désigne le déterminant de $\varphi$ agissant sur $D^{\prime}$ considéré comme $E_{w}$-espace vectoriel) et que

$$
t_{H}\left(D^{\prime}\right)=\frac{1}{\left[E_{w}: \mathbb{Q}_{p_{v}}\right]} \sum_{i \in \mathbb{Z}} i \operatorname{dim}_{F_{v}}\left(\mathrm{Fil}^{i} D_{F_{v}}^{\prime} / \mathrm{Fil}^{i+1} D_{F_{v}}^{\prime}\right)
$$

en notant $D_{F_{v}}^{\prime}=D^{\prime} \otimes_{F_{v}, 0} F_{v}$ et $\mathrm{Fil}^{i} D_{F_{v}}^{\prime}=D_{F_{v}}^{\prime} \cap \mathrm{Fil}^{i} D_{F_{v}}$ (on a normalisé $t_{N}\left(D^{\prime}\right)$ et $t_{H}\left(D^{\prime}\right)$ pour qu'ils soient invariants par extension des scalaires de $E_{w}$ et coïncident avec les définitions usuelles si $E_{w}=\mathbb{Q}_{p_{v}}$ et on note que ce ne sont plus nécessairement des entiers).

Soit $D_{F_{v}}=\bigoplus_{\sigma} D_{F_{v}, \sigma}$ la décomposition associée à l'isomorphisme (18). Chaque $D_{F_{v}, \sigma}$ est un $E_{w}$-espace vectoriel filtré, dont on note $\left(\nu_{\sigma}^{1}, \ldots, \nu_{\sigma}^{r}\right)$ est le $r$-uplet des pentes de Hodge (avec $\nu_{\sigma}^{1} \leq \cdots \leq \nu_{\sigma}^{r}$ ), qui est tel que

$$
\operatorname{dim}_{E_{w}} \mathrm{Fil}_{\sigma}^{k}=\sharp\left\{j, \nu_{\sigma}^{j} \geq k\right\}
$$

si on note ... Fil $\sigma_{\sigma}^{k} \supset \mathrm{Fil}_{\sigma}^{k+1} \ldots$ la filtration sur $D_{F_{v}, \sigma}$ vérifiant $\mathrm{Fil}^{k}=\bigoplus_{\sigma} \mathrm{Fil}_{\sigma}^{k}$. Soit $\nu$ la moyenne des $\nu_{\sigma}$ où $\sigma$ parcourt les plongements de $F_{v}$ dans $E_{w}$. 
Soit $D=\bigoplus_{\tau} D_{\tau}$ la décomposition associée à l'isomorphisme (19). Alors $D_{\tau}$ est un $E_{w}$-espace vectoriel de dimension $r, \varphi^{s}: D \rightarrow D$ préserve $D_{\tau}$ et agit de façon $E_{w}$-linéaire sur $D_{\tau}$ pour tout $\tau$, et le polynôme caractéristique de l'action de $\varphi^{s}$ sur $D_{\tau}$ est indépendant de $\tau$ (parce que $\varphi^{s}$ commute à $\varphi$ qui permute circulairement les $D_{\tau}$ ). On note c la classe de conjugaison semi-simple dans $G L_{r}\left(E_{w}\right)$ qui correspond à ce polynôme.

Alors $N_{\mathfrak{p}}(c) \leq \nu$, c'est-à-dire que $N_{\mathfrak{p}}(c)$ appartient à l'enveloppe convexe dans $X_{*}(\hat{T}) \otimes_{\mathbb{Z}} \mathbb{R}=\mathbb{R}^{r}$ des $w(\nu)$ pour $w \in \mathfrak{S}_{n}$.

Démonstration. - On remarque simplement, comme le fait Fontaine dans la preuve de la proposition 4.3.3. de [18], que tout point de rupture du polygone de Newton détermine un sous- $F_{v, 0} \otimes_{\mathbb{Q}_{p_{v}}} E_{w}$-module $D^{\prime}$ de $D$ stable par $\varphi$ (et donc libre) et comme

$$
v_{\mathfrak{p}}\left(\operatorname{det}_{E_{w}}\left(\left.\varphi^{s}\right|_{D_{\tau}^{\prime}}\right)\right)=v_{\mathfrak{p}}\left(\operatorname{det}_{E_{w}}\left(\left.\varphi\right|_{D^{\prime}}\right)\right)=t_{N}\left(D^{\prime}\right)
$$

ce point de rupture est $\left(\operatorname{rang}_{F_{v, 0} \otimes_{\mathbb{Q}_{p}} E_{w}} D^{\prime}, t_{N}\left(D^{\prime}\right)\right)$. Comme $\nu$ est la moyenne des $\nu_{\sigma}$ où $\sigma$ parcourt les plongements de $F_{v}$ dans $E_{w}$, pour tout sous- $F_{v, 0} \otimes \mathbb{Q}_{p_{v}} E_{w}$-module $D^{\prime}$ stable par $\varphi$ (et donc libre),

$$
\left(\operatorname{rang}_{F_{v, 0} \otimes_{\mathbb{Q}_{p_{v}}} E_{w}} D^{\prime}, t_{H}\left(D^{\prime}\right)\right)
$$

est au-dessus du polygone associé à $\nu$. Grâce à la condition de faible admissibité $t_{N}\left(D^{\prime}\right) \geq t_{H}\left(D^{\prime}\right)$ donc a fortiori le point de rupture

$$
\left(\operatorname{rang}_{F_{v, 0} \otimes \mathbb{Q}_{p_{v}} E_{w}} D^{\prime}, t_{N}\left(D^{\prime}\right)\right)
$$

est au-dessus du polygone associé à $\nu$.

On renvoie à [33] pour des résultats sur les polygones de Newton des isocristaux.

Comparons maintenant les estimées des propositions 3.1 et 4.1. Soit $M$ un motif de rang $r$ sur $F$ à coefficients dans $\overline{\mathbb{Q}}$ correspondant à $\pi|\operatorname{det}|^{\alpha}$ avec $\alpha \in$ $\frac{r-1}{2}+\mathbb{Z}$ et $\pi$ une forme automorphe cuspidale dont la partie finie apparait, via la formule de Matsushima, dans

$$
\lim _{\longrightarrow} H^{\bullet}\left(G L_{r}(F) \backslash G L_{r}(\mathbb{A}) / K K_{\infty}, W\right) \otimes_{E} \overline{\mathbb{Q}}
$$

où la limite est prise sur $K$ de plus en plus petit et où $W$ est comme dans le paragraphe 3 . On sait que $M$ doit avoir pour $r$-uplets de Hodge indexés par $i \in\{1, \ldots, n\}$ les $\nu_{i}=\rho+\mu_{i}-\alpha(1,1, \ldots, 1)$. En effet les $-w_{0}\left(\nu_{i}\right)$ sont les caractères infinitésimaux des composantes à l'infini de $\pi|\operatorname{det}|^{\alpha}$ (voir [11] et [37] pour des rappels sur les caractères infinitésimaux) et d'après les conjectures habituelles les $\nu_{i}$ sont les $r$-uplets de Hodge de $M$. Les classes de conjugaison de «Hecke » et de « Frobenius » dans $G L_{r}(\overline{\mathbb{Q}})$ associées à $\pi$ et $M$ comme dans les propositions 3.1 et 4.1 sont reliées par la puissance $\alpha^{\text {ième }}$ du cardinal du corps résiduel. Donc les estimées des propositions 3.1 et 4.1 se correspondent. 


\section{Cas des groupes non déployés, et lien avec un article de Dat}

Pour simplifier nous n'avons considéré dans cet article que des groupes déployés. En fait les mêmes arguments que ci-dessus donnent des estimées en toutes les places «non ramifiées déployées », c'est-à-dire en les places $v$ où le groupe $G$ s'étend en un groupe réductif déployé (encore noté $G$ ) sur l'anneau des entiers $\theta_{F_{v}}$ du corps local $F_{v}$ associé, et telles que le sous-groupe compact en $v$ soit conjugué à $G\left(\Theta_{F_{v}}\right)$. En les autres places non ramifiées des estimées existent aussi mais pour les justifier il faudrait reprendre l'article de Gross [23] dans le cadre non déployé (comme dans les paragraphes 1.11,1.12, 1.13 de [1], mais de façon plus explicite et avec des coefficients dans $\mathbb{Z}$ au lieu de $\mathbb{C}$ ).

Dans [13], Jean-François Dat introduit la notion de représentation $p$-adique localement entière pour des groupes $p$-adiques. Dans les notations du troisième paragraphe, si $G$ est un groupe réductif sur un corps de nombre $F, K_{\infty}$ un compact maximal à l'infini, si le système local est trivial (c'est-à-dire si $W$ est la représentation triviale), si $v$ est une place de $F$ au-dessus d'un nombre premier $p$, si $\pi$ est une forme automorphe pour $G$, telle que sa partie finie $\pi_{f}=\bigotimes_{w}$ place finie de ${ }_{F} \pi_{w}$ soit définie sur $\mathbb{Q}$ (pour simplifier, en général une extension finie de $\mathbb{Q}$ serait nécessaire) et que $\pi_{f}^{K^{v}}$ soit non nulle et apparaisse dans

$$
\lim _{\longrightarrow} H^{\bullet}\left(G(F) \backslash G(\mathbb{A}) / K^{v} K_{v} K_{\infty}, \mathbb{Q}\right)
$$

(où $K^{v}$, sous-groupe compact hors $v$, est fixe et la limite est prise sur $K_{v}$, sousgroupe compact en $v$, lorsqu'il devient de plus en plus petit), alors pour tout choix de $K_{v}$ l'intersection de $\pi_{f}^{K^{v} K_{v}} \otimes_{\mathbb{Q}} \mathbb{Q}_{p}$ et de

$$
\bar{H}^{\bullet}\left(G(F) \backslash G(\mathbb{A}) / K^{v} K_{v} K_{\infty}, \mathbb{Z}_{p}\right)
$$

est un $\mathbb{Z}_{p}$-réseau de $\pi_{f}^{K^{v} K_{v}} \otimes_{\mathbb{Q}} \mathbb{Q}_{p}$ stable par l'action de la $\mathbb{Z}_{p}$-algèbre de Hecke en $v$ relative à $K_{v}$ (c'est-à-dire la $\mathbb{Z}_{p}$-algèbre des fonctions sur $K_{v} \backslash G\left(F_{v}\right) / K_{v}$ à valeurs dans $\mathbb{Z}_{p}$, munie de la convolution pour la mesure de Haar sur $G\left(F_{v}\right)$ telle que $K_{v}$ ait pour masse 1). La représentation $\pi_{v} \otimes_{\mathbb{Q}} \mathbb{Q}_{p}$ est donc localement entière au sens de la définition 3.1 de [13] et Dat a classifié ces représentations, ce qui redonne nos estimées lorsque $G$ est déployé en $v$, donne certainement les bonnes estimées aux places non ramifiées non deployés, et donne encore des renseignements aux places ramifiées.

Pour $G=G L_{r}$ nous allons comparer les renseignements donnés par les résultats de [13] sur le paramètre de Langlands de la représentation localement entière $\pi_{v} \otimes_{\mathbb{Q}} \mathbb{Q}_{p}$ en une place ramifiée $v$, où $\pi$ est comme ci-dessus, avec ceux que fournit la faible admissibilité du $\left(\varphi, N, \operatorname{Gal}\left(L / F_{v}\right)\right)$-module filtré associé à un motif $M$ relié à $\pi$ par la correspondance de Langlands pour $G L_{r}$. Ce qui suit figure essentiellement dans [26]. 
Soit $W D\left(F_{v}\right)$ le groupe de Weil-Deligne de $F_{v}$. Pour chaque représentation $\tau$ de $W D\left(F_{v}\right)$ à coefficients dans $\overline{\mathbb{Q}}_{p}$ (muni de la valuation $v_{\mathfrak{p}}$ normalisée par $v_{\mathfrak{p}}\left(q_{v}\right)=1$ ) on appelle polygone de $\tau$ le polygone convexe ayant pour pentes $v_{\mathfrak{p}}\left(\alpha_{1}\right), \ldots, v_{\mathfrak{p}}\left(\alpha_{r}\right)$ où $\alpha_{1}, \ldots, \alpha_{r}$ sont les valeurs propres de n'importe quel relevé du Frobenius géométrique dans le groupe de Weil de $F_{v}$. On choisit une place $\mathfrak{p}$ de $\overline{\mathbb{Q}}$ et une place $v$ de $F$ au-dessus du même nombre premier $p$. Le choix de $\mathfrak{p}$ détermine un plongement $\overline{\mathbb{Q}} \rightarrow \overline{\mathbb{Q}}_{p}$. Soit $M$ un motif sur $F$ à coefficients dans $\overline{\mathbb{Q}}$, d'où une représentation de $\operatorname{Gal}(\bar{F} / F)$ à coefficients dans $\overline{\mathbb{Q}}_{p}$. La restriction de cette représentation à $\operatorname{Gal}\left(\bar{F}_{v} / F_{v}\right)$ est potentiellement semistable et la théorie de Fontaine lui associe un $\left(\varphi, N, \operatorname{Gal}\left(L / F_{v}\right)\right)$-module filtré faiblement admissible, où $L$ est une extension finie de $F_{v}$, ainsi qu'une représentation $\tau$ de $W D\left(F_{v}\right)$ à coefficients dans $\overline{\mathbb{Q}}_{p}$ (voir [21, 20, 26]). Comme dans la preuve du lemme 4.2 (ou comme dans le théorème 1.2 de [26]) la faible admissibité entraîne que le polygone de $\tau$ est au-dessus du polygone associé à $\rho$ (la demi-somme des racines positives de $G L_{r}$ ). En effet comme $\pi_{f}^{K^{v}}$ apparaît dans la cohomologie du sytème local trivial, pour tout plongement de $F$ dans $\overline{\mathbb{Q}}$, le polygone de Hodge du motif $M$ relié à $\pi$ doit être le polygone associé à $\rho$. L'équivalence entre (ii) et (iii) du théorème 1.2 de [26] montre que la condition de faible admissibilité ne donne pas d'autre renseignement sur le polygone de $\tau$ (en dehors du fait qu'il est au-dessus du polygone associé à $\rho$ ).

D'autre part la proposition suivante est une réinterprétation de la conjecture 3.8 de [13], que Jean-François Dat démontre pour $G L_{r}$.

Proposition 5.1. - Soit $\pi_{v}$ une représentation lisse admissible et irréductible de $G L_{r}\left(F_{v}\right)$ à coefficients dans $\overline{\mathbb{Q}}_{p}$ et $\tau$ la représentation de $W D\left(F_{v}\right)$ qui lui est associée par la correspondance de Langlands locale. Alors $\pi_{v}$ est localement entière si et seulement si le polygone de $\tau$ est au-dessus du polygone associé à $\rho$ et a les mêmes extrémités ( $\rho$ est la demi-somme des racines positives de $\left.G L_{r}\right)$.

Donc dans le cas d'un système local trivial on obtient exactement les mêmes estimées pour les formes automorphes cuspidales cohomologiques pour $G L_{r}$ et les motifs qui leur correspondent (conjecturalement). La remarque 3.17 de [13] suggère que cela devrait rester vrai pour les systèmes locaux arbitraires (voir aussi l'implication (i) $\Rightarrow$ (ii) du théorème 1.2 de [26]).

\section{Comparaison avec les estimées des normes archimédiennes des valeurs propres de Hecke}

La notion de représentation $p$-adique localement entière est l'analogue $p$ adique de la notion de représentation complexe bornée (i.e. dont tous les coefficients de matrice sont bornés). Ces représentations sont bien comprises (cf [8]),

TOME $139-2011-\mathrm{N}^{\mathrm{O}} 4$ 
et pour les représentations sphériques, la classification des fonctions sphériques bornées fait intervenir l'enveloppe convexe des $w(\rho)$.

On suppose $G$ semi-simple pour simplifier. On pourrait donc obtenir des estimées des normes archimédiennes des valeurs propres de Hecke analogues aux estimées $p$-diques qui font l'objet de cet article en utilisant le fait que les représentations automorphes cuspidales sont bornées en tant que représentations complexes. Cependant ces estimées archimédiennes n'ont aucun intérêt car elles sont beaucoup plus grossières que celles résultant du fait que les représentations automorphes cuspidales sont unitaires, et infiniment plus grossières que les estimées proposées par les conjectures de Ramanujan-Petersson (qui sont reliées par la correspondance de Langlands aux conjectures de Weil mentionnées au début de l'introduction).

Cependant les estimées $p$-adiques des propositions 2.1 et 3.1 sont optimales pour les corps de fonctions (pour $G L_{r}$ et pour les autres groupes si on admet la correspondance de Langlands), et très probablement pour les corps de nombres.

\section{BIBLIOGRAPHIE}

[1] D. Blasius \& J. D. Rogawski - «Zeta functions of Shimura varieties », in Motives (Seattle, WA, 1991), Proc. Sympos. Pure Math., vol. 55, Amer. Math. Soc., 1994, p. 525-571.

[2] A. BorEL - «Properties and linear representations of Chevalley groups », in Seminar on Algebraic Groups and Related Finite Groups (The Institute for Advanced Study, Princeton, N.J., 1968/69), Lecture Notes in Math., vol. 131, Springer, 1970, p. 1-55.

[3] _ « Stable real cohomology of arithmetic groups », Ann. Sci. École Norm. Sup. 7 (1974), p. 235-272.

[4] « and Lie groups (Notre Dame, Ind., 1980), Progr. Math., vol. 14, Birkhäuser, 1981, p. 21-55.

[5] _ , Regularization theorems in Lie algebra cohomology. Applications », Duke Math. J. 50 (1983), p. 605-623. Correction and complement, Duke Math. J., 60 (1990), 299-301.

[6] A. Borel \& L. JI - Compactifications of symmetric and locally symmetric spaces, Mathematics : Theory \& Applications, Birkhäuser, 2006.

[7] A. Borel \& J-P. SERRE - « Corners and arithmetic groups », Comment. Math. Helv. 48 (1973), p. 436-491.

[8] A. Borel \& N. R. WAllaCH - Continuous cohomology, discrete subgroups, and representations of reductive groups, Annals of Math. Studies, vol. 94, Princeton Univ. Press, 1980. 
[9] P. CARTIER - «Representations of $p$-adic groups : a survey », in Automorphic forms, representations and L-functions (Proc. Sympos. Pure Math., Oregon State Univ., Corvallis, Ore., 1977), Part 1, Proc. Sympos. Pure Math., XXXIII, Amer. Math. Soc., 1979, p. 111-155.

[10] C. Chevalley - «Certains schémas de groupes semi-simples », in Séminaire Bourbaki, vol. 6, Soc. Math. France, 1995, Séminaire Bourbaki, Vol. 1960/61, exposé n 219 , p. 219-234.

[11] L. ClOzEL - «Motifs et formes automorphes : applications du principe de fonctorialité », in Automorphic forms, Shimura varieties, and L-functions, Vol. I (Ann Arbor, MI, 1988), Perspect. Math., vol. 10, Academic Press, 1990, p. 77-159.

[12] _ _ « Cours à l'institut Tata », 1994.

[13] J.-F. DAT - «Représentations lisses $p$-tempérées des groupes $p$-adiques », Amer. J. Math. 131 (2009), p. 227-255.

[14] P. Deligne - «La conjecture de Weil. I», Publ. Math. I.H.É.S. 43 (1974), p. 273-307.

[15] — « La conjecture de Weil. II », Publ. Math. I.H.É.S. 52 (1980), p. $137-252$.

[16] P. Deligne \& J-P. Serre - «Formes modulaires de poids $1 »$, Ann. Sci. École Norm. Sup. 7 (1974), p. 507-530.

[17] M. Demazure \& A. Grothendieck - Schémas en groupes. III : Structure des schémas en groupes réductifs, Lecture Notes in Math., vol. 153, 1964.

[18] J.-M. Fontaine - « Modules galoisiens, modules filtrés et anneaux de Barsotti-Tate », in Journées de Géométrie Algébrique de Rennes. (Rennes, 1978), Vol. III, Astérisque, vol. 65, Soc. Math. France, 1979, p. 3-80.

[19] _ _ 《aleurs spéciales des fonctions $L$ des motifs », Astérisque 206 (1992), p. 205-249, Séminaire Bourbaki, Vol. 1991/92, exposé nº 751.

[20] _ _ «eprésentations $l$-adiques potentiellement semi-stables », Astérisque 223 (1994), p. 321-347.

[21] _ _ «eprésentations p-adiques semi-stables », Astérisque 223 (1994), p. $113-184$.

[22] J.-M. Fontaine \& B. Perrin-Riou - « Autour des conjectures de Bloch et Kato : cohomologie galoisienne et valeurs de fonctions $L »$, in Motives (Seattle, WA, 1991), Proc. Sympos. Pure Math., vol. 55, Amer. Math. Soc., 1994, p. 599-706.

[23] B. H. Gross - « On the Satake isomorphism », in Galois representations in arithmetic algebraic geometry (Durham, 1996), London Math. Soc. Lecture Note Ser., vol. 254, Cambridge Univ. Press, 1998, p. 223-237. 
[24] H. HIDA - « Control theorems of $p$-nearly ordinary cohomology groups for $\mathrm{SL}(n) »$, Bull. Soc. Math. France 123 (1995), p. 425-475.

[25] _ GL $(n) »$, Mém. Soc. Math. Fr. (N.S.) 67 (1996).

[26] Y. Hu - « Normes invariantes et existence de filtrations admissibles », J. reine angew. Math. 634 (2009), p. 107-141.

[27] J. C. JANTZEN - Representations of algebraic groups, second éd., Mathematical Surveys and Monographs, vol. 107, Amer. Math. Soc., 2003.

[28] L. Lafforgue - «Chtoucas de Drinfeld et correspondance de Langlands », Invent. Math. 147 (2002), p. 1-241.

[29] R. P. LANGLANDs - «Modular forms and $\ell$-adic representations », in $M o$ dular functions of one variable, II (Proc. Internat. Summer School, Univ. Antwerp, Antwerp, 1972), Lecture Notes in Math., vol. 349, Springer, 1973, p. 361-500.

[30] D. Mauger - «Algèbres de Hecke quasi-ordinaires universelles », Ann. Sci. École Norm. Sup. 37 (2004), p. 171-222.

[31] B. Mazur - « Frobenius and the Hodge filtration», Bull. Amer. Math. Soc. 78 (1972), p. 653-667.

[32] « «robenius and the Hodge filtration (estimates)», Ann. of Math. 98 (1973), p. 58-95.

[33] M. RApoport \& M. Richartz - « On the classification and specialization of F-isocrystals with additional structure », Compositio Math. 103 (1996), p. 153-181.

[34] P. Schneider \& J. Teitelbaum - « Banach-Hecke algebras and $p$-adic Galois representations », Doc. Math. Extra Vol. (2006), p. 631-684.

[35] J. SCHWERMER - « Cohomology of arithmetic groups, automorphic forms and $L$-functions », in Cohomology of arithmetic groups and automorphic forms (Luminy-Marseille, 1989), Lecture Notes in Math., vol. 1447, Springer, 1990, p. 1-29.

[36] J-P. SERRE - « Groupes de Grothendieck des schémas en groupes réductifs déployés », Publ. Math. I.H.É.S. 34 (1968), p. 37-52.

[37] R. TAYLOR - «Galois representations », Ann. Fac. Sci. Toulouse Math. 13 (2004), p. 73-119.

[38] D. A. J. Vogan \& G. J. ZUCKERMAN - « Unitary representations with nonzero cohomology », Compositio Math. 53 (1984), p. 51-90. 